\title{
IMPEDANCE-BASED WIRELESS SENSOR NETWORK FOR METAL-PROTECTIVE COATING EVALUATION
}

\author{
A Thesis \\ Submitted to the Graduate Faculty \\ of the \\ North Dakota State University \\ of Agriculture and Applied Science
}

\begin{abstract}
By
Ronghua Yu

In Partial Fulfillment of the Requirements

for the Degree of

MASTER OF SCIENCE
\end{abstract}

Major Department:

Electrical and Computer Engineering

August 2011

Fargo, North Dakota 


\section{North Dakota State University \\ Graduate School}

Title

Impedance-based Wireless Sensor Network for

Metal-protective Coating Evaluation

By

Ronghua Yu

The Supervisory Committee certifies that this disquisition complies with North Dakota State University's regulations and meets the accepted standards for the degree of

North Dakota State University Libraries Addendum

To protect the privacy of individuals associated with the document, signatures have been removed from the digital version of this document. 


\section{ABSTRACT}

Ronghua Yu, M.S., Department of Electrical and Computer Engineering, College of Engineering and Architecture, North Dakota State University, August 2011. Impedance-based Wireless Sensor Network for Metal-Protective Coating Evaluation. Major Professor: Chao You.

Research has focused on the influences of flowing fluid on the corrosion of bare metals, but there is little emphasis on the degradation of metal-protective coating. Evaluating the metal-protective coating usually uses the Electrochemical Impedance Spectroscopy (EIS) method. This paper presents a new impedance-based wireless sensor network for metal-protective coating evaluation. This wireless sensor network consists of two parts: impedance-based wireless sensor nodes and a wireless data base that are equipped with a network analyzer (AD5933) and a RF transceiver (CC1111/CC1110). In the experiment, three coating panels are immersed in flowing deionized water (DI water) and one coating panel immersed in stationary DI water. Experimental results demonstrate that this wireless sensor network is capable of evaluating the coating degradation.

Keywords: Impedance, frequency, AD5933, coating evaluation, deionized water (DI water). 


\section{ACKNOWLEDGEMENTS}

This thesis is first and foremost dedicated to my parents, who have devoted their lives to me and are always my strongest supporters.

I wish to express my love and gratitude to my beloved spouse, YuanYuan Zhou, for her unconditional understanding, support and endless love in all moments of our lives.

I wish to express my gratitude to my advisor, Dr. Chao You, who was abundantly helpful and offered invaluable assistance, support and guidance.

Deepest gratitude is also due to the members of the graduate committee, Dr. Benjamin Braaten, Dr. Srinivasan Sudarshan, and Dr. Yechun Wang without whose knowledge and assistance, this study would not have been successful. 


\section{TABLE OF CONTENTS}

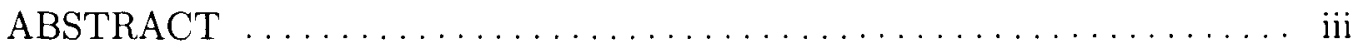

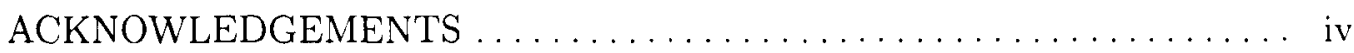

LIST OF TABLES $\ldots \ldots \ldots \ldots \ldots \ldots \ldots \ldots \ldots \ldots \ldots \ldots \ldots \ldots \ldots \ldots \ldots$

LIST OF FIGURES $\ldots \ldots \ldots \ldots \ldots \ldots \ldots \ldots \ldots \ldots \ldots \ldots \ldots \ldots$ viii

CHAPTER 1. INTRODUCTION $\ldots \ldots \ldots \ldots \ldots \ldots \ldots \ldots \ldots \ldots \ldots$

CHAPTER 2. BACKGROUND ..................... 4

2.1. Overview $\ldots \ldots \ldots \ldots \ldots \ldots \ldots \ldots \ldots \ldots \ldots \ldots \ldots \ldots \ldots \ldots \ldots \ldots \ldots, 4$

2.2. EIS Coating Evaluation Method $\ldots \ldots \ldots \ldots \ldots \ldots \ldots \ldots \ldots$

2.3. ENM Coating Evaluation Method $\ldots \ldots \ldots \ldots \ldots \ldots \ldots$

2.4. Other Coating Evaluation Methods .................. 7

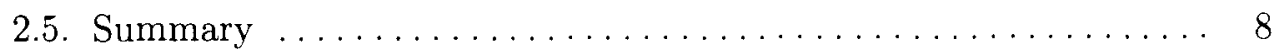

CHAPTER 3. PROPOSED IMPEDANCE-BASED WIRELESS SENSOR

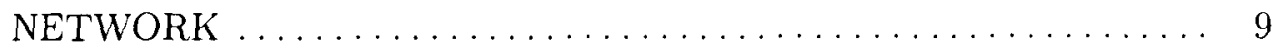

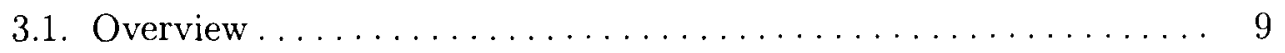

3.2. IWSN Hardware Design $\ldots \ldots \ldots \ldots \ldots \ldots \ldots \ldots \ldots \ldots$

3.2.1. AD5933 Characteristics and Operation ........... 10

3.2.2. CC1111/CC1110 Characteristics and Operation....... 13

3.2.3. Comparison of AD5933 and Gamry Impedance Analyzer . 14

3.3. IWSN Software Design and Operation ............... 17

3.4. IWSN Power Requirements and Consumption ............ 20

CHAPTER 4. EXPERIMENTS AND RESUITS .............. 22 
4.1. Overview ............................... 22

4.2. Coating Panel Material and Preparation ............... 23

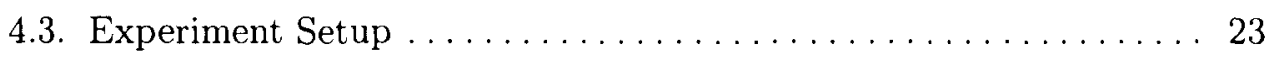

4.3.1. Flowing Immersion Setup ................ 24

4.3.2. Stationary Immersion Setup ............... 26

4.4. Experiment Results....................... 27

4.4.1. Experiment Results of Flowing Immersion $\ldots \ldots \ldots \ldots 27$

4.4.2. Experiment Results of Stationary Immersion ......... 31

4.5. Experiment Summary .......................... 32

CHAPTER 5. CONCLUSION AND FUTURE WORK $\ldots \ldots \ldots \ldots \ldots 34$

5.1. Accomplishments........................... 34

5.2. Contributions ............................ 34

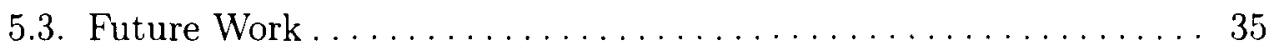

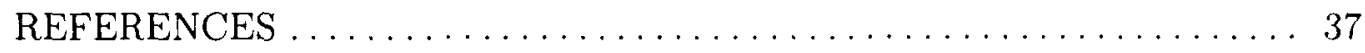

APPENDIX A. SOURCE CODE FOR THE WIRELESS SENSOR NODE . 40 APPENDIX B. SOURCE CODE FOR THE WIRELESS DATA BASE $\ldots .48$ 


\section{LIST OF TABLES}

Table

$\underline{\text { Page }}$

$1 \quad$ Impedance Analyzer Comparison $\ldots \ldots \ldots \ldots \ldots \ldots \ldots \ldots$

2 Wireless Sensor Node Voltage and Current Requirements . . . . . . . . 20

3 Wireless Sensor Node Power Requirements . . . . . . . . . . . . . 21 


\section{LIST OF FIGURES}

Figure

$\underline{\text { Page }}$

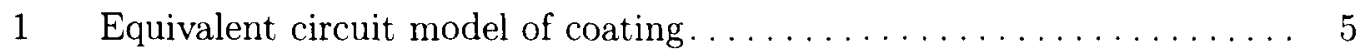

2 Typical ENM 3 -electrode cell configuration ................ 7

$3 \quad$ Impedance-based wireless data base $\ldots \ldots \ldots \ldots \ldots \ldots \ldots \ldots$

$4 \quad$ Impedance-based wireless sensor nodes $\ldots \ldots \ldots \ldots \ldots \ldots \ldots \ldots$

5 The pin configuration of AD5933 $[10] \ldots \ldots \ldots \ldots \ldots \ldots \ldots \ldots \ldots \ldots$

$6 \quad$ Functional block diagram of AD5933 $[10] \ldots \ldots \ldots \ldots \ldots \ldots \ldots, 12$

7 The pin configuration of $\mathrm{CC} 1111 / \mathrm{CC} 1110 \ldots \ldots \ldots \ldots \ldots \ldots \ldots$

8 Application circuit for $\mathrm{CC} 1111 / \mathrm{CC} 1110$ with the fundamental crytal .. 15

$9 \quad$ Gamry Reference 600 impedance analyzer .................. 15

10 Wireless sensor node $\ldots \ldots \ldots \ldots \ldots \ldots \ldots \ldots \ldots \ldots \ldots \ldots \ldots$

11 Additional circuit for small impedance measurement $\ldots \ldots \ldots \ldots \ldots 18$

12 Wireless star topology $\ldots \ldots \ldots \ldots \ldots \ldots \ldots \ldots \ldots \ldots \ldots \ldots \ldots$

13 Flow chart of wireless data base and sensor nodes . . . . . . . . . . 19

14 Graphic user interface of wireless sensor network ............ 20

15 Coating sample................................. 23

16 Experimental setup of flowing immersion $\ldots \ldots \ldots \ldots \ldots \ldots \ldots 25$

17 Schematic diagram of flowing immersion $[27] \ldots \ldots \ldots \ldots \ldots \ldots 25$

18 Experimental setup of stationary immersion $\ldots \ldots \ldots \ldots \ldots \ldots \ldots 26$

19 Impedance modulus as a function of frequency at different immersion days for panel \#1 measured by EIS $\ldots \ldots \ldots \ldots \ldots \ldots \ldots \ldots \ldots$ 
20 Impedance modulus as a function of frequency at different immersion days for panel \#1 measured by IWSN

21 Impedance modulus as a function of frequency at different immersion days for panel \#2 measured by EIS

22 Impedance modulus as a function of frequency at different immersion days for panel \#2 measured by IWSN . . . . . . . . . . . . . . 30

23 Comparison of coating impedance measured by EIS and IWSN for panel

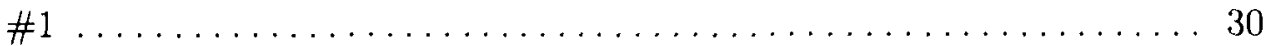

24 Comparison of coating impedance measured by EIS and IWSN for panel \#2

25 Impedance modulus as a function of frequency at different immersion days for panel \#4 measured by EIS

26 Impedance modulus as a function of frequency at different immersion days for panel \#4 measured by IWSN . . . . . . . . . . . . . . 33

27 Comparison of coating impedance measured by EIS and IWSN for panel $\# 4$ 


\section{CHAPTER 1. INTRODUCTION}

Metal-protective coating is widely used in the protection of structures, such as pipelines, bridges and other metal materials [1]. The failure of paints and coatings generates a large amount of financial loss every year. The annual cost of corrosion for the United States of America is estimated around $\$ 300$ billion and approximately $\$ 3$ billion is attributed to military aircraft system for corrosion costs [3].

Metal-protective coating corrosion happens when the coating is penetrated by moisture, ions and oxygen from the environment. Moisture is an important factor for metal protective coating degradation, mostly resulting in cathodic delamination and blistering near a coating defect. The cathodic delamination and blistering lead to advanced damages, such as fracture and deformation. These structural damages are both potential financial and life threats. In order to reduce or prevent these potential threats, over the past few decades research has been dedicated to detecting, monitoring and preventing coating corrosion.

Research in coating evaluation has been implemented since a long time ago and has yielded some achievements. However, most traditional coating evaluations based on the EIS have to use the impedance analyzers, such as the Reference 600 Potentiostat provided by Gamry Instruments. This kind of analyzer is expensive (around $\$ 40,000)$ and impractical to carry in the field. Therefore, it is necessary to develop a portable and convenient coating evaluation instrument for metal-protective coating in the practical field based on EIS. Emma Angelini developed a handheld impedance measurement system [4] that can be used for electrochemical measurement. However, it can only realize the one point measurement. In addition, Emma used digital signal processor (DSP) as the main controller and designed the peripheral circuits, such as low pass filter, digital-to-analog converter (DAC) and calibration circuit. Nevertheless, the circuit will be more stability and consume less power if there is a 
chip that includes all the blocks required for impedance evaluation. Analog Devices, a chip company, has made an impedance analyzer chip called AD5933 that can realize a portable, miniaturized and multi-positions monitoring impedance analyzer network. For example, in [22] a new radio-frequency (RF) sensing node is proposed to replace the traditional impedance-based structural health monitoring method in loose bolt inspection application. The sensing node consists of an AD5933, a micro-controller and a RF transmitter. However, the author used the AD5933 development board as the sensor node and the size is around $10 \mathrm{~cm} \times 8 \mathrm{~cm}$. Besides the AD5933 development board, the author also used another micro-controller development board to control the AD5933 development board. The equipment designed by the author is too bulky to carry, especially for the field application. In addition, there is only one sensor node used in the application that is not satisfied to the multi-position monitoring.

This paper presents a new Impedance-based Wireless Sensor Network (IWSN) for metal-protective coating evaluation, which is mainly realized by an impedance analyzer (AD5933) and $\mathrm{RF}$ transceiver (CC1111/CC1110). It is a miniaturized impedance analyzer retwork that can be controlled at a PC terminal by the serial communication. The advantages of AD5933 are low power consumption and integration of most blocks for impedance spectroscopy. The impedance measurement range of $\mathrm{AD} 5933$ is from $1 \mathrm{~K} \Omega$ to $10 \mathrm{M} \Omega$ and the excitation frequency is ranged from $1 \mathrm{KHz}$ to $100 \mathrm{KHz}$. In order to measure small impedance of coating, an additional circuit is adopted in this network. The $\mathrm{RF}$ transceiver $\mathrm{CC} 1111 / \mathrm{CC} 1110$ is a true low-power sub-1 GHz System-On-Chip (SoC) designed for low-power wireless applications and it is responsible for controlling AD5933 and wireless communication [8]. The working current of IWSN operates around $25 \mathrm{~mA}$ during the measurement and the power consumption is only $30 \mu \mathrm{W}$ when sensor nodes are in sleeping mode. IWSN can realize the portable wireless impedance analysis for metal-protective coating evaluation in the 
field with the advantages of low power consumption, compact size and user friendly interface operation. 


\section{CHAPTER 2. BACKGROUND}

\subsection{Overview}

Metal-protective coating that is designed to extend the lifetime of metal structures. The performance of coatings usually depends on their properties. However, the metal-protective coating can be effected by corrosions with the consequence that the metal beneath the coating is unprotected. Therefore, the monitoring and reduction of the corrosion rate of a metal structure is an important topic.

Traditionally, there are several coating electrochemical evaluation methods, such as EIS, Electrochemical Noise Method (ENM) and DC resistance [13]. ENM is usually used to identify corrosion while EIS is mainly used to predict coating remaining life as mentioned in the published papers $[9,26]$.

\subsection{EIS Coating Evaluation Method}

EIS is a rapid and convenient technique well suited for evaluating coating permeability or barrier properties based on the electrical resistance of the coating. The impedance of a coating is related to the nature of the polymer, its density, and its fillers. EIS has been used widely in the laboratory for determining coating performance and for obtaining quantitative kinetic and mechanistic information on coating deterioration.

The impedance of a coating is observed to decrease as a function of time of exposure to a corrosive environment. The decrease in impedance is observed to be related to the loss of barrier properties and the onset of under-film corrosion. In laboratory studies of various generic types of coatings, the relative performance of different coatings could be determined well in advance of the visible effects of deterioration; these studies also showed a good relationship between coating impedance and coating field performance [13]. 
Metal-protective coatings and electrochemical corrosion processes both have an inherent resistance and capacitance characters. AC signal can identify and quantitatively measure the individual resistance and capacitance elements. The coating system can be modeled as an equivalent circuit model based on EIS data. Fig. 1 illustrates the model of coating. The model [2] contains the elements of coating capacitance $\left(\mathrm{C}_{c}\right)$, double layer capacitance $\left(\mathrm{C}_{d l}\right)$, solution resistance $\left(\mathrm{R}_{s}\right)$, coating resistance $\left(\mathrm{R}_{c t}\right)$, and pore resistance $\left(\mathrm{R}_{p o}\right)$. This model is called a Randles Cell, which is one of the simplest and most common cell models. When the coating is degraded, the composition of the resistance and the capacitance will change. The variation of capacitive component reflects the phase change. The variation of resistive component reflects the change of coating impedance.

Traditionally, the frequency range applied by EIS is from $0.01 \mathrm{~Hz}$ to $100 \mathrm{KHz}$. The typical Root Mean Square (R.M.S) values of imposed voltage might be $100 \mathrm{mV}$ or $10 \mathrm{mV}$ and the data is demonstrated as Nyquist (linear) Bode (log) plots. The inspection of the Bode plot readily provides very useful information on coating performance. High-performance coatings with good barrier properties behave as pure capacitors with a simple, readily recognized response. A higher frequency generates a lower impedance. Generally, the coating with impedance greater than $10^{9} \Omega / \mathrm{cm}^{2}$ at $0.1 \mathrm{~Hz}$ is considered to perform well for corrosion protection [13]. On the other hand, the coating with impedance less than $10^{6} \Omega / \mathrm{cm}^{2}$ at $0.1 \mathrm{~Hz}$ is considered to be in poor protection condition.

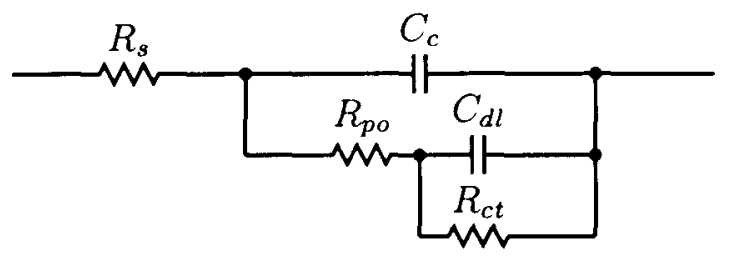

Figure 1. Equivalent circuit model of coating 


\subsection{ENM Coating Evaluation Method}

Currently, EIS is one of the most commonly reproduced methods for evaluation of coating corrosion protective properties and it is considered as the electrochemical studies of coatings. However, this technique depends on externally imposed perturbing signal to the system and requires relatively expensive electrochemical instrumentation.

ENM, as an electrochemical measurement method, has existed since the 1970s [7] and it has been used for over 25 years in basic electrochemistry. The ENM technique was first applied to coating evaluation during the late 1980s and early 1990s $[5,26]$ and it has been proved remarkably successful. At that time, Skerry and co-workers used ENM to examine organic coating failure and since this date ENM seems to be an easier method than EIS to apply and to use. This method was followed by Bierwagen et al $[6,17]$. From then, a wide variety of coating evaluations used ENM because ENM is not intrusive and it is easily automatable.

The ENM theory is to measure the fluctuations of current and potential noise that is generated by sudden film rupture, crack propagation and metal deformation. The noise comes from various sources, such as thermal noise, shot noise and flicker noise. In the metal-protective coating applications, the electrochemical noise originates in part from certain type of corrosion processes, especially in localized corrosion phenomena, and is often regarded as a random phenomenon related to deterministic corrosion kinetics. The electrochemical noise can be used to provide an indication of the rate and stability of the corrosion process. This technique doesn't require the external signal. Because of this property, the ENM coating evaluation is also a non-intrusive and non-destructive evaluation (NDE) technique.

The typical ENM 3-electrode cell configuration is shown in Fig. 2. There are two working electrodes (WE) and a reference electrode (RE). However, it needs two 
nominally identical specimens which is a practical disadvantage because the noise signal between the two identical specimens is influenced by the environment easily. It is necessary to use a shielding box to against the external signal. The traditional procedure is to immerse two identical coated electrodes in an electrolyte and monitor potential noise of the electrodes versus a reference electrode while simultaneously monitoring the current noise between the two electrodes [16]. The full details on how to apply ENM to coating evaluation are given in $[18,19]$.

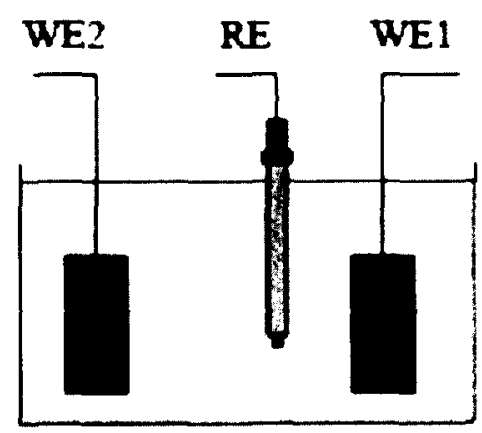

Figure 2. Typical ENM 3-electrode cell configuration

\subsection{Other Coating Evaluation Methods}

Other electrochemical methods have been invented for coating evaluation. In the 1950s, Bacon et al evaluated 300 coating systems in sea water by using a resistance measuring device. They found coating systems with $100 \Omega / \mathrm{cm}^{2}$ had good protection, and those between $1 \Omega / \mathrm{cm}^{2}$ and $100 \Omega / \mathrm{cm}^{2}$ had fair performance. In $1973, \mathrm{Dr}$ Mayne's laboratory in Cambridge observed the discrete anodes and cathodes on polystyrene that means resistance control was important, and an instrument called Keithley electrometer was invented for the easier coating evaluation [20]. This coating 
evaluation method is the same as the DC resistance method that is used today. In 2000, Fu et al. proposed a method in evaluating coating failure by using acoustic emission [12]. In 2002, Sekin et al. used the electrochemical, physiochemical and physical measurements to evaluate the degradation of the coating corrosion [23]. In 2005, Simmers presented a method by using beams to detect coating corrosion. Nevertheless, all of these methods need bulky and expensive equipment, and they cannot implement the wireless multi-position monitoring and control.

\subsection{Summary}

This Chapter discussed the traditional methods for monitoring and evaluating metal-protective coatings. EIS and ENM are the most popular methods used in coating evaluation. Although EIS, ENM and DC resistance are useful in coating evaluation, there are still many disadvantages. The equipment based on those methods are bulky, expensive and inconvenient. Therefore, it is necessary to design a portable coating evaluation system. In Chapter 3 , a new impedance-based coating evaluation system is proposed. It has many advantages, such as wireless, multi-position, user friendly interface and low power consumption. 


\section{CHAPTER 3. PROPOSED IMPEDANCE-BASED WIRELESS SENSOR NETWORK}

\subsection{Overview}

The proposed IWSN consists of two parts: impedance-based wireless sensor nodes and a wireless data base. The wireless sensor node contains an impedance measurement chip AD5933 and a RF transceiver CC1111/CC1110.

The wireless sensor node measures the coating impedance and sends that data to the wireless data base. The wireless data base contains a RF transceiver CC1111/CC1110 that is used to receive data from wireless sensor nodes and to send data to the PC terminal by using serial communication. Both the wireless sensor nodes and the base station are powered by two AA $1.5 \mathrm{~V}$ batteries. The block diagrams are shown in Fig. 3 and Fig. 4.

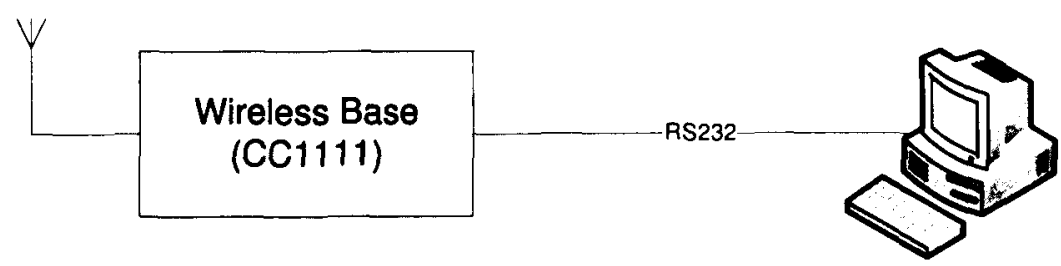

Figure 3. Impedance-based wireless data base

\subsection{IWSN Hardware Design}

The IWSN is equipped with the impedance measurement single chip AD5933 and the RF Transceiver CC1111/CC1110. It is a miniaturized wireless impedance measurement network that can be controlled at a PC terminal by using serial communication. The advantages of IWSN are portablity, low power consumption and low price. 


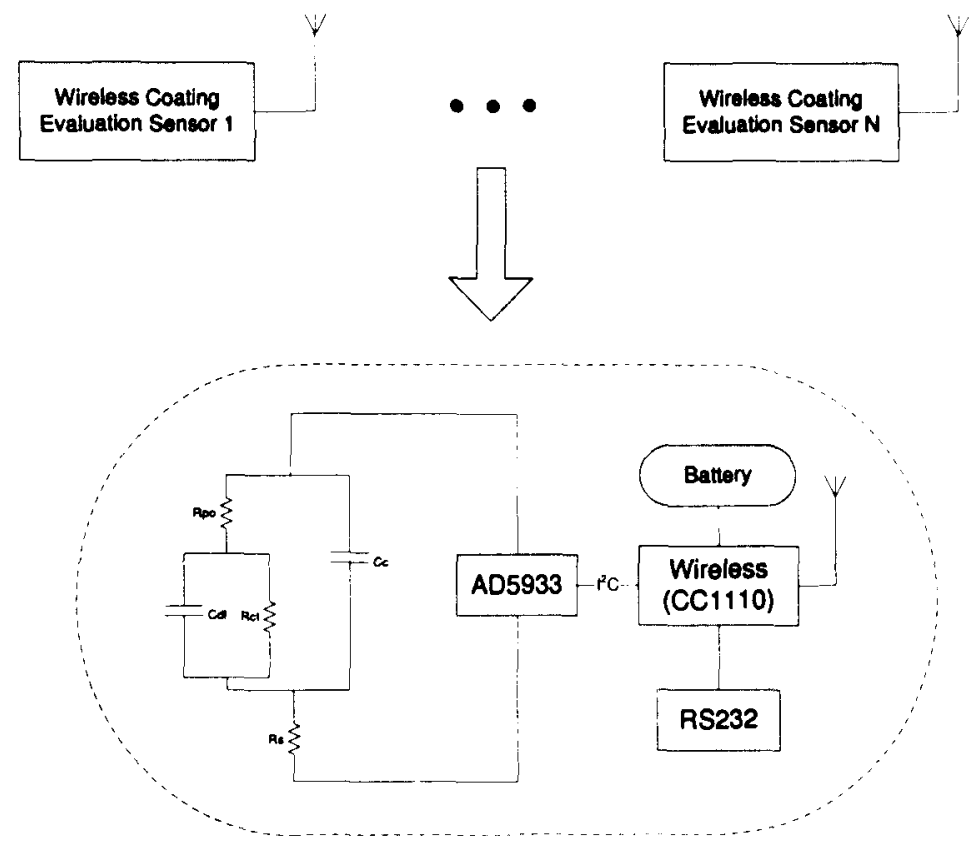

Figure 4. Impedance-based wireless sensor nodes

\subsubsection{AD5933 Characteristics and Operation}

AD5933 is a high precision impedance converter developed by Analog Devices. It combines an on-board frequency generator with a 12-bit, 1 mega sample per second (MSPS), analog-to-digital converter (ADC). The chips under consideration for this system are the AD5933 and the AD5934. These chips are nearly identical in operation, power requirements, and features. The only difference between the two chips is that the AD5933 has a 1MSPS sampling rate, and the AD5934 has a $250 \mathrm{kSPS}$ sampling rate. The higher sampling rate of the AD5933 leads to a slightly higher cost of $\$ 6.65$ per chip rather than $\$ 4.35$ for an AD5934. The pin configuration is shown in Fig. 5. The cost difference has been deemed negligible for this application considering the higher performance that can be achieved with the AD5933. The remainder of the work in this thesis has been performed with the AD5933.

The AD5933 has most blocks required for EIS, such as an A/D converter, a 


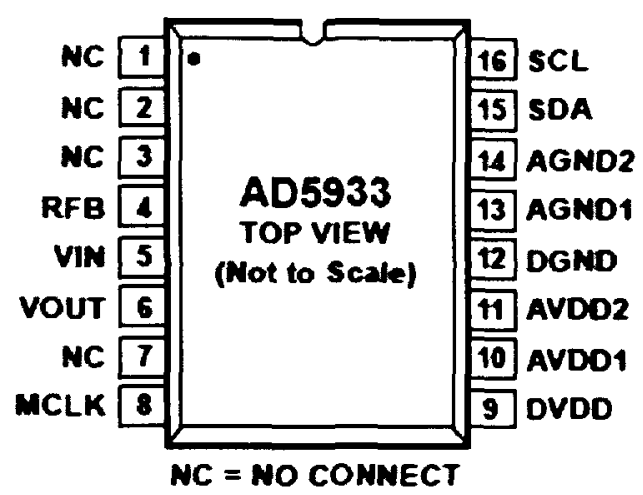

Figure 5. The pin configuration of AD5933 [10]

Discrete Fourier Transform (DFT), a signal generator [10]. The detailed functional block diagram is shown in Fig. 6. There are two signal paths inside AD5933. One path generates the excitation sinusoidal signal with a known frequency to excite the external complex impedance. It contains a Direct Digital Synthesis (DDS), a D/A converter, an operational amplifier with programmable gain and an output resistance. The other signal path, called measurement path, is used to measure the response signal generated from the previous path. The signal is passed through a low-pass filter and sampled by a 12-bits A/D converter. The converted data will go through the DFT and DFT algorithm will generate both a real (R) and imaginary (I) dataword at each frequency point along the sweep [10]. The impedance and phase of coating are calculated by using following equations:

$$
\begin{gathered}
\text { Magnitude }=\sqrt{R^{2}+I^{2}} \\
\text { Phase }=\tan ^{-1}(I / R)
\end{gathered}
$$

The general operation of the chip can be outlined as follows. A 27-bit DDS core 


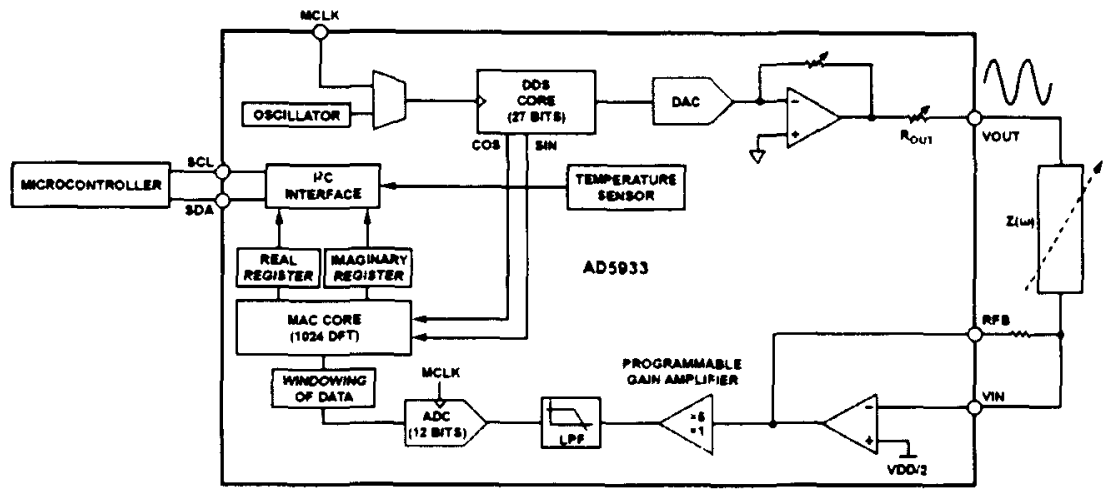

Figure 6. Functional block diagram of AD5933 [10]

produces a digitized sine wave of the desired interrogation frequency. The excitation signal is then passed through a programmable gain stage and output into the device of interest. The current output from the device caused by the excitation signal is then passed through a current-to-voltage amplifier, which features a user-selectable feedback resistor in order to vary the amplifier gain. The amplifier feedback resistor must be sized appropriately in order to ensure that the signal remains in the linear range of the analog-to-digital converter (ADC). The output from the current to voltage amplifier is then sent through a low-pass anti-aliasing filter and sent to a 12-bit, 1-MSPS ADC. A Hanning window is applied to the digital data, and a 1024 point $\mathrm{DFT}$ is performed at the frequency point of interest. The resulting real and imaginary values of the DFT are passed to a micro-controller in twos complement format. This procedure is repeated for every point in the desired frequency sweep.

The user can perform the frequency sweep with a user-defined start frequency, frequency resolution, and number of points in the sweep. The peak-to-peak value of excitation signal applied to the external unknown impedance between the VOUT and VIN pins can also be programmed.

The magnitude of impedance should be calculated firstly by using Equation (1) before calculating the impedance. After getting the magnitude of impedance, it must 
be multiplied by a scaling factor called the gain factor. The gain factor is generated when the system is calibrated with a known impedance connected between the VOUT and VIN pins. The calculation of any unknown impedance between the VOUT and VIN pins can be implemented based on the magnitude and gain factor stated above, see Equation (3).

$$
\text { Unknown Impedance }=\frac{1}{\text { Gain Factor } * \text { Magnitude }}
$$

The measured impedance range of AD5933 is from $1 \mathrm{~K} \Omega$ to $10 \mathrm{M} \Omega$ and the output frequency range is from $1 \mathrm{KHz}$ to $100 \mathrm{KHz}$. However, AD5933 has finite frequency responses. The gain factor also varies with frequency. The variation of gain factor will result in errors in the impedance calculation. In order to reduce the error, the range of frequency should be limited to a narrow range.

\subsubsection{CC1111/CC1110 Characteristics and Operation}

The RF transceiver $\mathrm{CC} 1111 / \mathrm{CC} 1110$ is a low-power sub-1 $\mathrm{GHz}$ system-onchip (SoC) that combines the excellent performance of the RF transceiver CC1101 with an industry-standard enhanced 8051 Micro Controller Unit (MCU) [15]. The pin configuration is shown in Fig. 7. In this design, CC1111/CC1110 communicate with $\mathrm{AD} 5933$ by using $\mathrm{I}^{2} \mathrm{C}$ protocol. $\mathrm{I}^{2} \mathrm{C}$ is a multi-master serial singleended computer bus invented by Philips. However, the CC1111/CC1110 doesn't have $\mathrm{I}^{2} \mathrm{C}$ interface and it has to emulate the $\mathrm{I}^{2} \mathrm{C}$ protocol by using general $\mathrm{I} / \mathrm{O}$ to communicate with AD5933. After the establishment of a connection between AD5933 and $\mathrm{CC} 1111 / \mathrm{CC} 1110, \mathrm{CC} 1111 / \mathrm{CC} 1110$ can write and read the internal registers of AD5933 to set up the parameters stated above, such as start frequency, frequency increment, and number of increments.

After setting up all the parameters of AD5933, AD5933 measures the coating impedance. The $\mathrm{CC} 1111 / \mathrm{CC} 1110$ reads the coating impedance data and sends them 


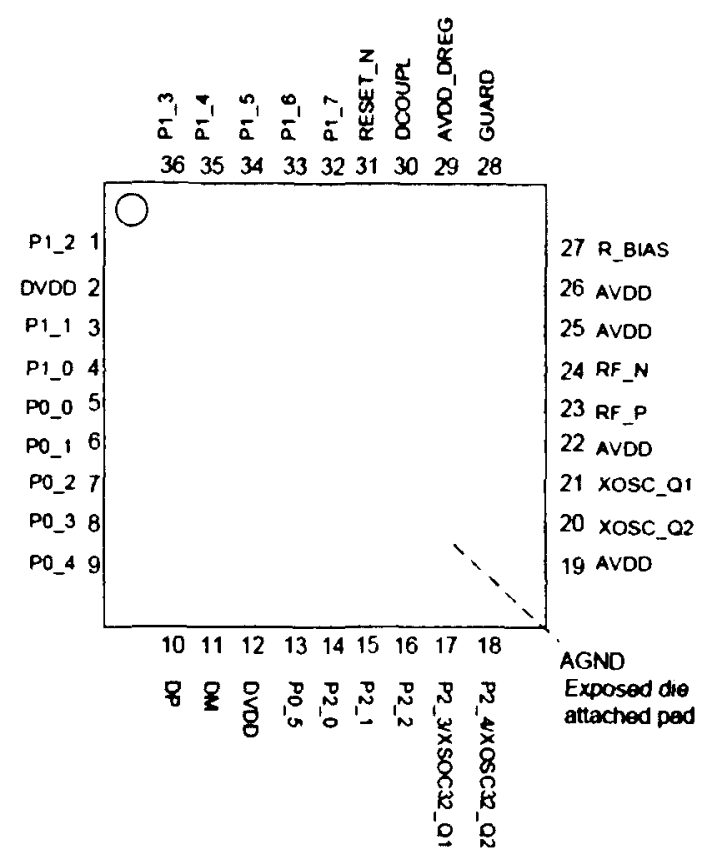

Figure 7. The pin configuration of $\mathrm{CC} 1111 / \mathrm{CC} 1110$

to the wireless data base by using wireless. The application circuit for CC1111/CC1110 is shown in Fig.. When coating impedance measurement is finished, the CC1111/CC1110 goes to the sleep mode in order to save energy until next measurement period. The application circuit for $\mathrm{CC} 1111 / \mathrm{CC} 1110$ is shown in Fig. 8.

\subsubsection{Comparison of AD5933 and Gamry Impedance Analyzer}

In order to illustrate the significance of this design by using AD5933, a comparison was made between a conventional Gamry Reference 600 Potentiostat impedance analyzer, Fig. 9, and the AD5933, Fig. 10. The performance characteristics of these two impedance analyzers are tabulated in Table 1.

From Table 1, the performance of Gamry Reference 600 Potentiostat impedance analyzer is superior to the performance of AD5933, but the AD5933 has the advantages of size, cost, power consumption and multi-position monitoring. For the practical application, coating evaluation usually needs a large array of sensor nodes 


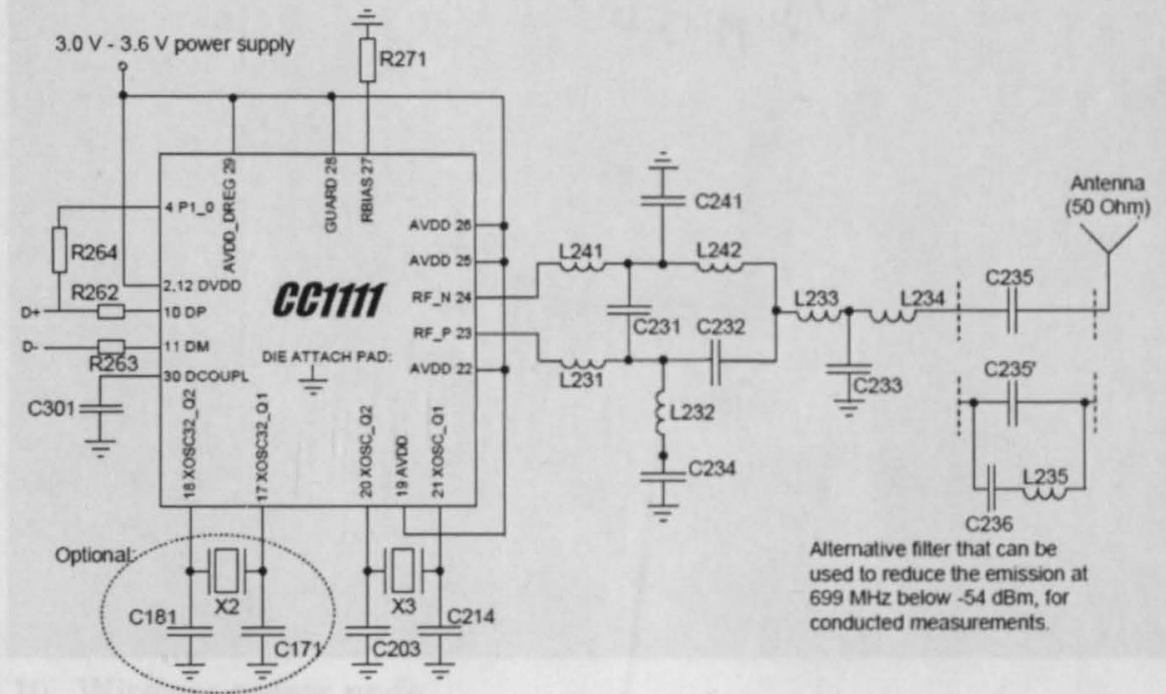

Figure 8. Application circuit for $\mathrm{CC} 1111 / \mathrm{CC} 1110$ with the fundamental crytal

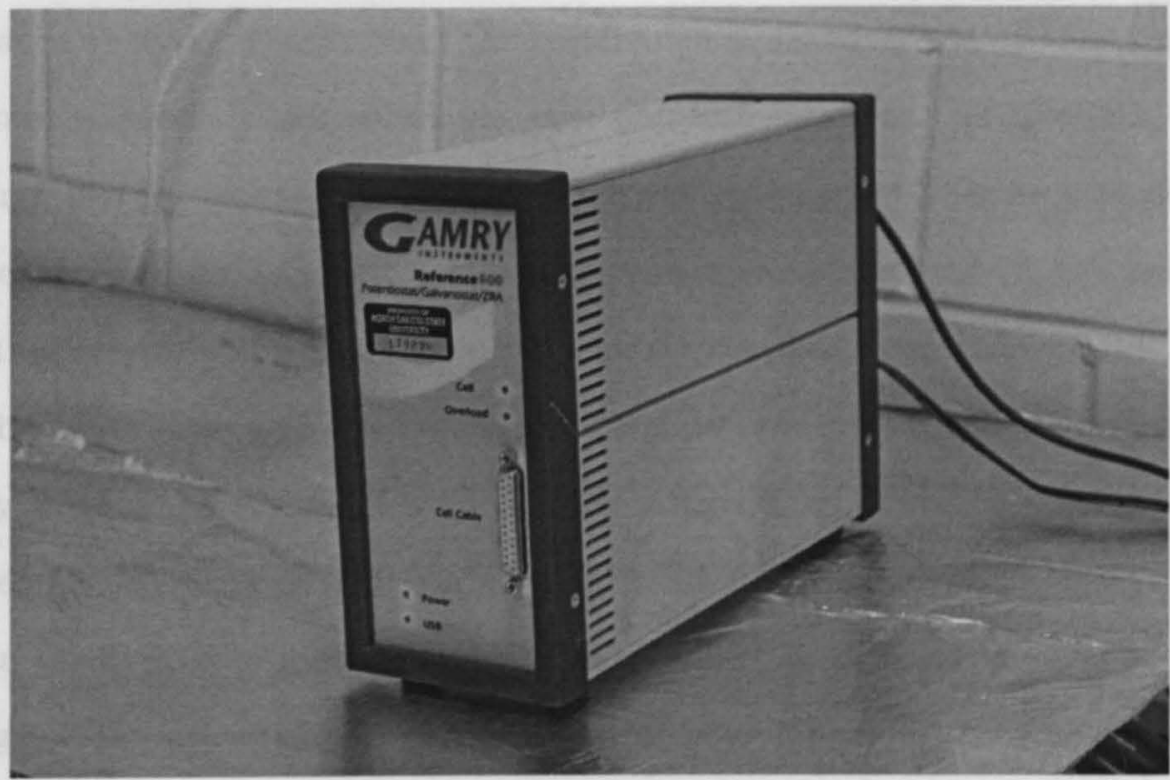

Figure 9. Gamry Reference 600 impedance analyzer 


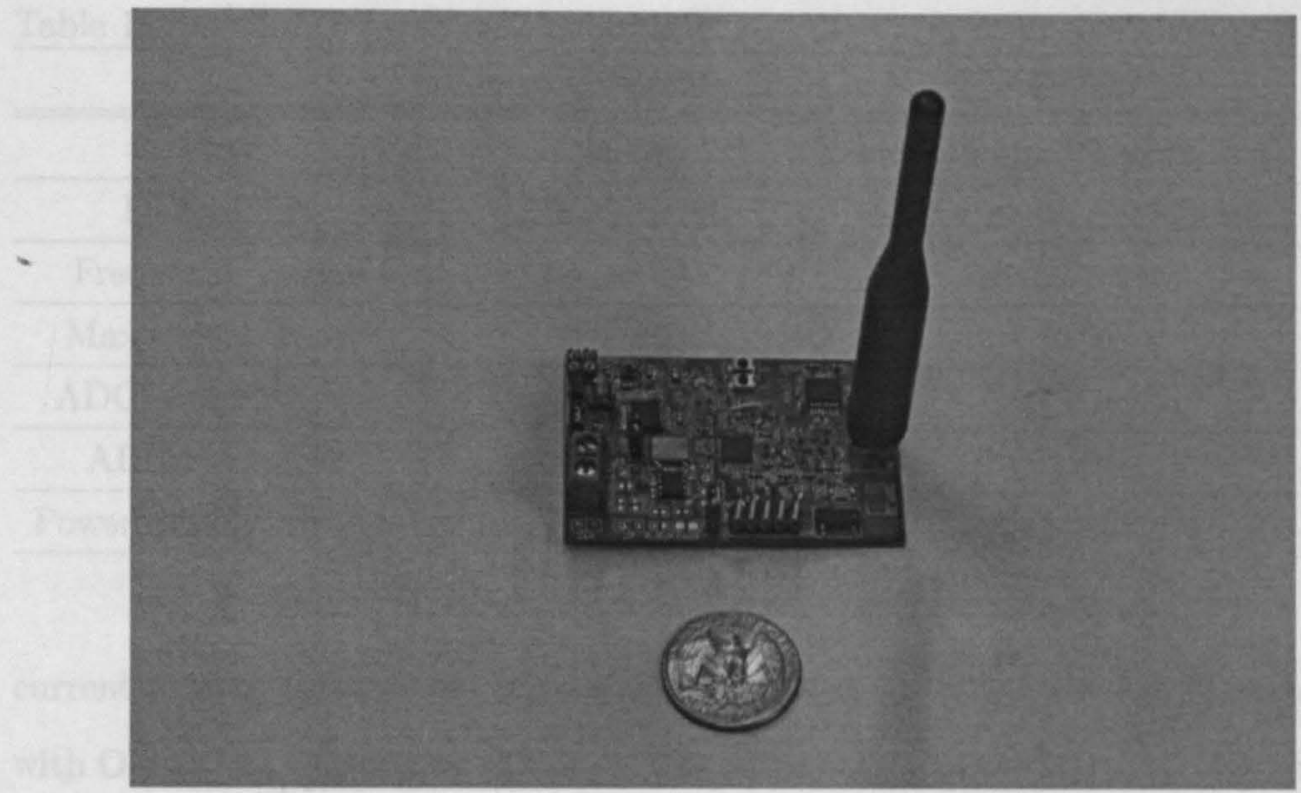

Figure 10. Wireless sensor node

distributed on a coated structure, such as pipeline. A large scale of deployment of coating evaluation requires the sensor nodes to be small, low power consuming, lightweight, and low cost. Obviously, the Gamry Reference 600 Potentiostat impedance analyzer doesn't meet the requirements. However, AD5933 is only $\$ 6.65$ and $6 \mathrm{~mm} \times 5 \mathrm{~mm}$, which solves the cost and size concerns. In addition, Gamry Reference 600 Potentiostat impedance analyzer requires $120 \mathrm{~V} \mathrm{AC}$ connections and wire deployment. It is inconvenient and expensive when applying Gamry equipment to coating evaluation on a structure. In contrast, a sensor node utilizing the AD5933 could be powered by two AA batteries. The data measured by sensor nodes can be sent by wireless, so it doesn't need the wire connections. Therefore, the AD5933 is designed for the portable impedance-based sensor node application.

In addition, the impedance range of AD5933 is also not enough for certain coating evaluations. In order to increase the range of impedance, an additional circuit is added in this system, shown in Fig. 11. If the user places a small impedance value $(\leq 500 \Omega)$ between the $V_{O U T}$ and $V_{I N}$ pins, it will result in an increase in signal 
Table 1. Impedance Analyzer Comparison

\begin{tabular}{c||c||c|c}
\hline & Gamry Reference 600 & AD5933 & Unit \\
\hline \hline Cost & 41000 & sensor node: 90, chip: 6.95 & $\$$ \\
\hline Dimensions & $270 \times 90 \times 190$ & sensor node: $60 \times 40 \times 12$ & $\mathrm{~mm}$ \\
\hline Frequency Range & $10 \mu$ to $1 \mathrm{M}$ & $1 \mathrm{~K}$ to $100 \mathrm{~K}$ & $\mathrm{~Hz}$ \\
\hline Max points/sweep & 262,143 & 512 & points \\
\hline ADC sampling rate & (at least) 200 & 1 & MSPS \\
\hline ADC resolution & 16 & 12 & bit \\
\hline Power Supply Voltage & $120 \mathrm{AC}$ & $3.3 \mathrm{DC}$ & $\mathrm{V}$ \\
\hline
\end{tabular}

current flowing through the impedance for a fixed excitation voltage in accordance with Ohm's law. According to Fig. 4, The output stage of the transmit side amplifier available at the $V_{O U T}$ pin may not be able to provide the required increase in current through the impedance. In order to have a unity gain condition about the receive side I-V amplifier, the user needs to have a similar value of feedback resistance for system calibration. Therefore, it is necessary to design a circuit that minimizes the effects of the issues stated above. In this design, an additional external amplifier circuit is placed along the signal path. The aim of the circuit is to place the AD5933 system gain within its linear range when measuring small impedances. The external amplifier attenuates the peak-to-peak excitation voltage at $V_{O U T}$ by a suitable choice of resistors (R1 and $R 4$ ), thereby reducing the signal current flowing through the impedance and minimizing the effect of the output series resistance in the impedance calculations [8].

\subsection{IWSN Software Design and Operation}

The IWSN uses the star topology that is based on the SimpliciTI protocol [25]. SimpliciTI is a simplified RF network solution developed by Texas Instruments in order to provide a wireless solution at low power, low cost and low data rate networks 


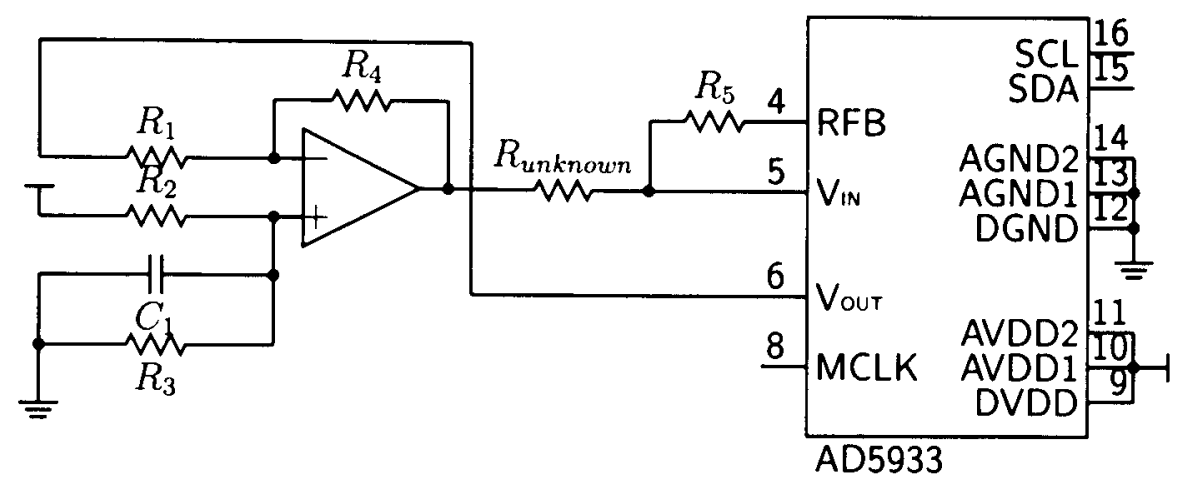

Figure 11. Additional circuit for small impedance measurement

for customers. The wireless star topology that is used in this application is shown in Fig. 12.

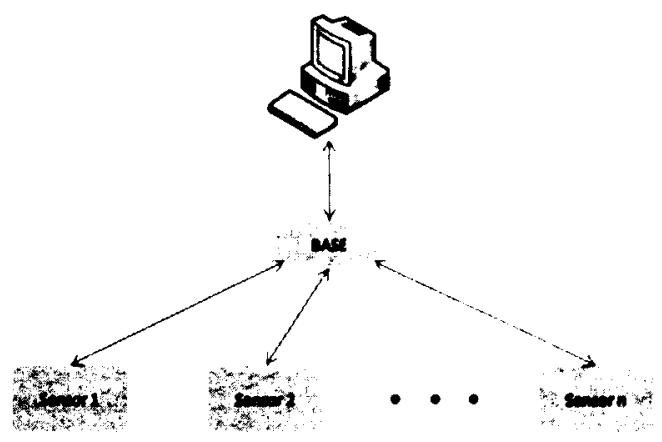

Figure 12. Wireless star topology

The PC communicates with wireless data base by using RS232. The wireless data base waits for the linking messages from wireless sensor. The wireless data base is responsible for storing and forwarding data collected from sensor nodes to the PC. If a wireless sensor joins the star network, it sends impedance data to the wireless data base and the wireless data base stores-and-forwards the data to the PC. The PC has a Visual Basic (VB) Graphic User Interface (GUI) and generates impedance graphs according to the received data. Every wireless sensor is assigned a period of time to be in sleeping mode in order to save power. At the end of the sleeping mode, the wireless sensor will be waken automatically and try to join the star network. After 
the establishment of a connection between the wireless data base and wireless sensor nodes, wireless sensor nodes will implement the impedance measurement and send data to the wireless data base. After the measurement, the wireless sensor returns to sleep mode until next period. The flow chart is shown in Fig. 13.
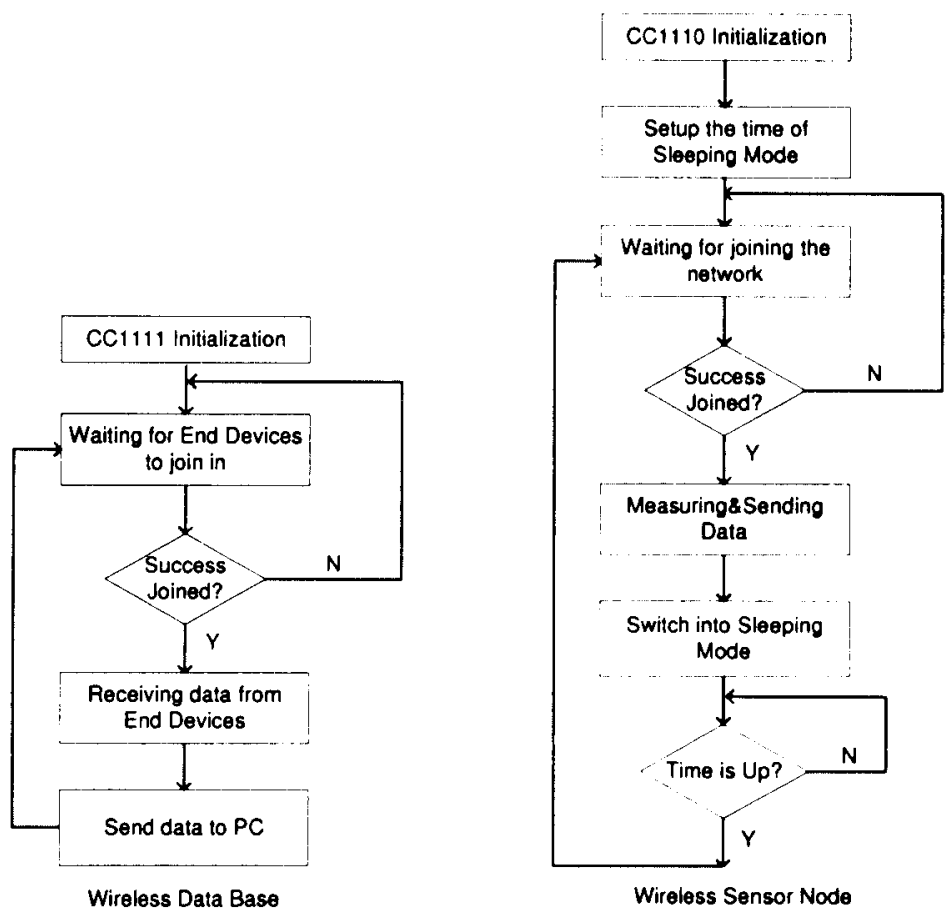

Figure 13. Flow chart of wireless data base and sensor nodes

The VB GUI is shown in Fig. 14. This is a user friendly interface and very convenient to operate. The user can setup parameters for frequency sweep, such as start frequency, frequency increment, and number of increments, according to different applications. The PC sends all setup parameters to the wireless data base when the user clicks the "Analysis" button, and then the wireless data base will send the parameters to wireless sensors. A wireless sensor will write the received parameters to the registers of $\mathrm{AD} 5933$ by $\mathrm{I}^{2} \mathrm{C}$ protocol and starts the impedance measurement. The user can also save pictures and data when the impedance analysis is finished just by clicking the button "Save Pics". 


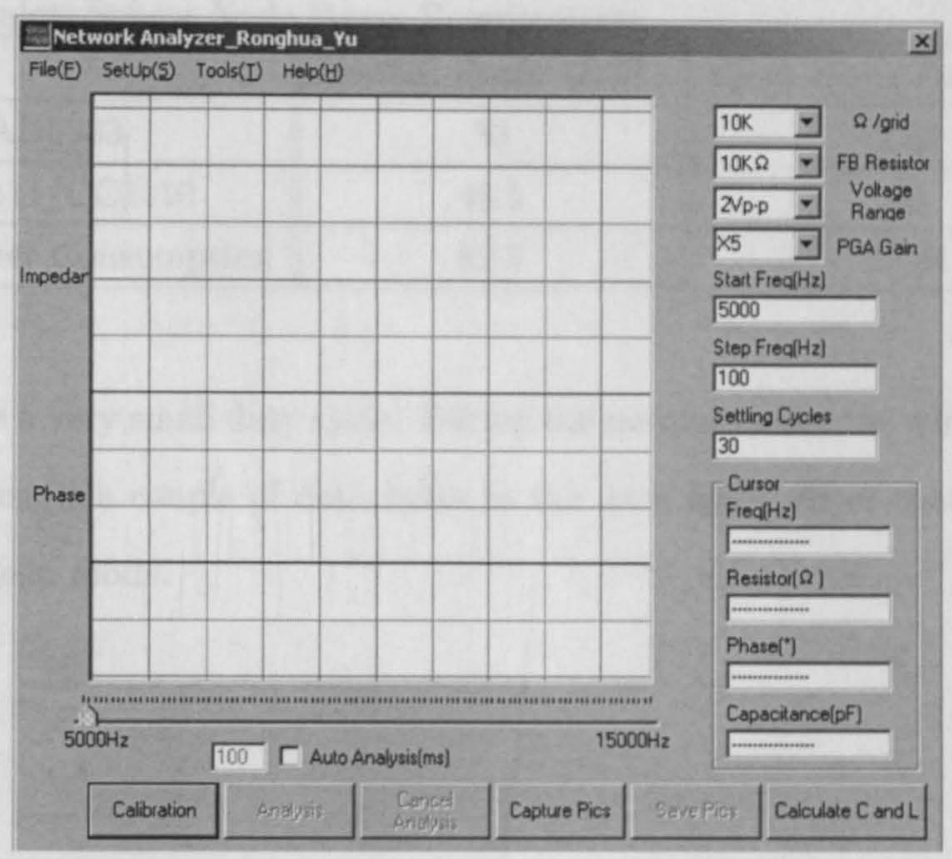

Figure 14. Graphic user interface of wireless sensor network

\subsection{IWSN Power Requirements and Consumption}

The power consumption of wireless sensor nodes should be considered carefully in order to save energy because all the wireless sensor nodes are powered by batteries. Table 2 shows the voltage and current requirements and consumption of various wireless sensor node components during both normal operation and sleep mode. The power requirements and consumptions are shown in Table 3

Table 2. Wireless Sensor Node Voltage and Current Requirements

\begin{tabular}{c||c||c||c}
\hline & Voltage Range (V) & Normal Current $(\mathrm{mA})$ & Sleep Mode Current $(\mu \mathrm{A})$ \\
\hline AD5933 & 3.3 & 10 & 0.7 \\
\hline CC1111/CC1110 & 3.3 & 15 & 0.5 \\
\hline Total Current & & 25 & 1.2 \\
\hline
\end{tabular}

From the power requirements and consumption table, it shows the CC111/CC1110 is the greatest power consumer in the wireless sensor node. Fortunately, the wireless 
Table 3. Wireless Sensor Node Power Requirements

\begin{tabular}{c||c||c}
\hline & Normal Power $(\mathrm{mW})$ & Sleep Mode Power $(\mu \mathrm{W})$ \\
\hline AD5933 & 33 & 2.31 \\
\hline CC1111/CC1110 & 49.5 & 30 \\
\hline Total Power Consumption & 82.5 & 32.31 \\
\hline
\end{tabular}

chip will have a very small duty cycle. During the normal mode, the wireless chip just needs to transmit a couple of data bytes to the data base. After that, the wireless chip enters sleep mode. 


\section{CHAPTER 4. EXPERIMENTS AND RESULTS}

\subsection{Overview}

This experiment was conducted out to demonstrate the impedance variation of coating under a frequency range. Flowing fluid may cause the abrasion of the coatings, enhance the supply of oxygen and diffusion or transport of ions, as a result of corrosion. Research has emphasized on the influences of flowing fluid on the corrosion of bare metals, but there is little attention to the degradation of metalprotective coatings [27]. In order to demonstrate the influence of flowing fluid on metal-protective coatings and verify the validity of IWSN, two different experimental setups were implemented: coating panels immersed in flowing deionized water (DI water) and coating panels immersed in stationary DI water. The stationary DI water setup is designed for comparison with the flowing DI water setup. There are three coating panels immersed in flowing DI water and one coating panel immersed in stationary DI water in this experiment. The duration time of this experiment is 30 days. Three panels ( \#1, \#2, and \#4) are selected to demonstrate the degradation of coating panels.

The wireless sensors nodes are connected to the counter electrodes and working electrodes of coating panels. Every sensor node measures the impedance of coating within a user-defined frequency range, generates the real part and imaginary part of the coating impedance and sends them to the wireless data base. The selected frequency range that applied to coating sample is from $1 \mathrm{KHz}$ to $30 \mathrm{KHz}$ and the frequency range is divided into four sections $(1 \mathrm{KHz}$ to $5 \mathrm{KHz}, 5 \mathrm{KHz}$ to $10 \mathrm{KHz}$, $10 \mathrm{KHz}$ to $20 \mathrm{KHz}$, and $20 \mathrm{KHz}$ to $30 \mathrm{KHz}$ ). The four frequency sections will be combined in the plot of the impedance modulus as a function of frequency. The PC plots the graph of impedance directly based on the data received by the wireless data base. 


\subsection{Coating Panel Material and Preparation}

The organic coatings used in this study are Korabor Aluminum primer RP140 and Korethan Topcoat UT6581 (K1), both supplied by KCC Corporation. The primer RP140 is a chlorinated rubber based coating with aluminum flake pigment. The topcoat is a polyurethane resin based finish coat. The stainless steel panels (Q-Panel Lab Products, Cleveland, $\mathrm{OH}$ ) are cut into $3 \times 3$ in squres and then penetrated by abrasion with sand paper and finally cleaned with hexane. The liquid paints are applied by air spraying and then dip coating is employed to protect the edges of the panel. Copper wire is attached onto the stainless steel panel before coatings are applied. 3M 764 tape is warpped around the edges and the back of the coated panel to protect the electrical connection and to create a well-defined exposure area. The coating sample is shown in Fig. 15.

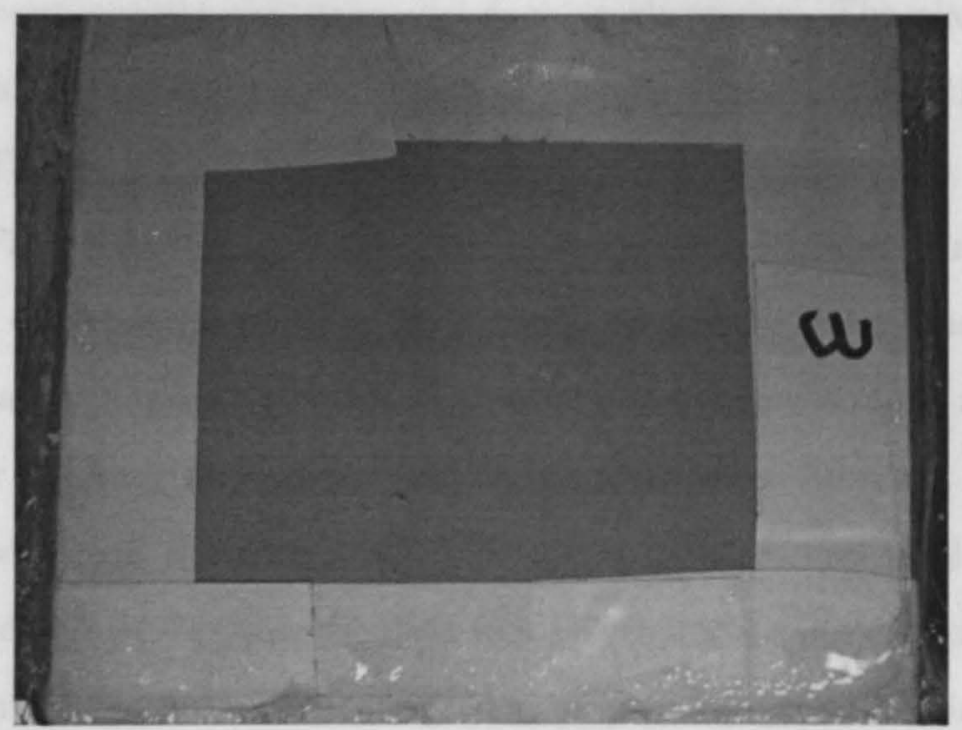

Figure 15. Coating sample

\subsection{Experiment Setup}

This experiment involves two setups. The first setup is the flowing immersion setup, and coating panels are immersed in the flowing DI water in order to emulate 
the influence of the flowing fluid environment. The second one is called stationary immersion setup. It is an O-ring glass electrochemical cell and it is filled with the DI water.

\subsubsection{Flowing Immersion Setup}

This experimental setup is adopted from the design of Wang and Bierwagen [27], which is shown in Fig. 16. The schematic diagram is shown in Fig. 17. The working DI water is stored in the fluid reservoir and pumped into the test parts. The DI water will be returned to the reservoir through the flow channel that is constructed with plastic sheets. The rotameter maintains the fluid flow rate at $3.683 \mathrm{~cm}^{3} / \mathrm{s}$ and the temperature is controlled at $77^{\circ} \mathrm{F}\left(25^{\circ} \mathrm{C}\right)$ by a water bath and a temperature control system. Three coated stainless steel panels are adhered to the upper and lower channel walls by a silicone rubber sealant (GE361). The flowing fluid goes through the gap between counter electrodes and working electrodes. The platinum meshes are embedded through the plastic plate to the upper channel wall acting as counter electrodes. The coated stainless steel panels act as working electrodes.

From Fig. 17, the wireless sensor nodes are connected to the counter electrodes and working electrodes to measure the coating impedance. Every sensor node measures the coating impedance within a user-defined frequency range and generates the real part and imaginary part of the coating impedance. After the measurement, wireless sensors send the data to the wireless data base. The wireless data base receives the data and forwards the data to a $\mathrm{PC}$ through serial communication. At the PC terminal, a user GUI is designed in order to generate coating impedance graphs according to the received data. In this experiment, the selected frequency range that is applied to coating panels is from $1 \mathrm{KHz}$ to $30 \mathrm{KHz}$, and the frequency range is divided into four sections. The four frequency sections will be combined when the PC plots the impedance modulus as a function of frequency. 


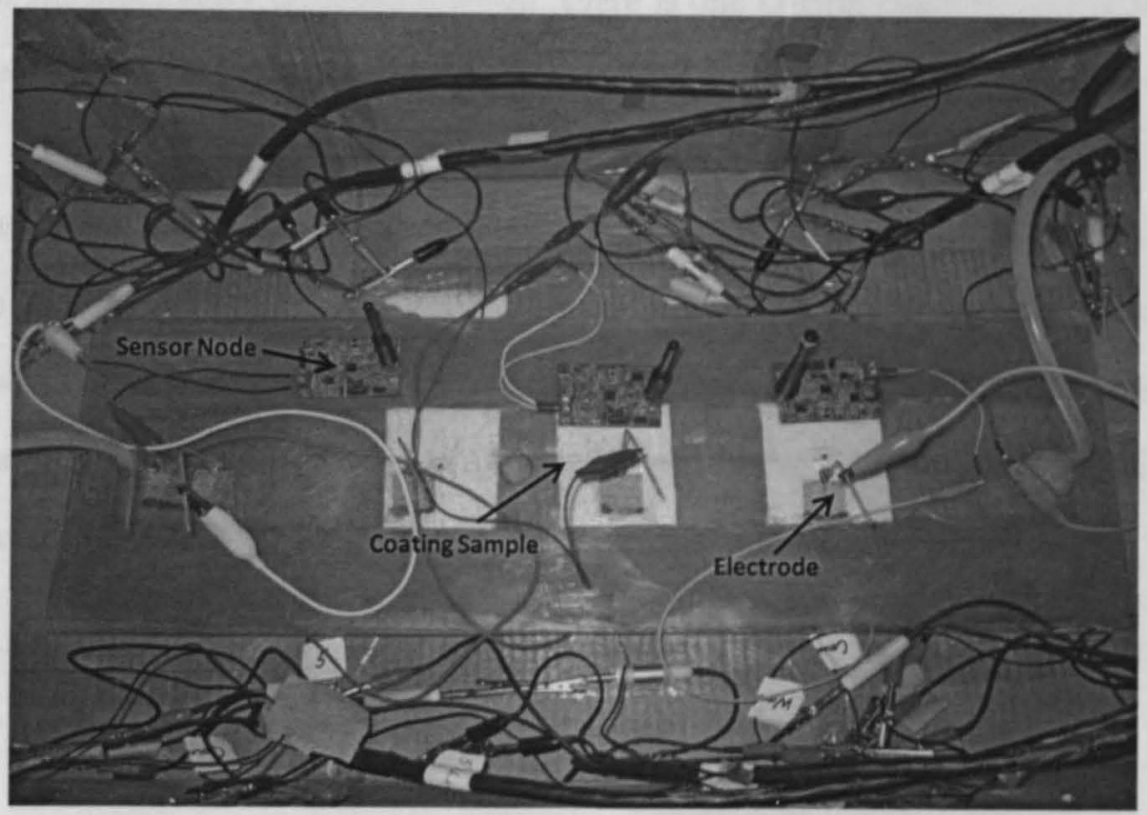

Figure 16. Experimental setup of flowing immersion

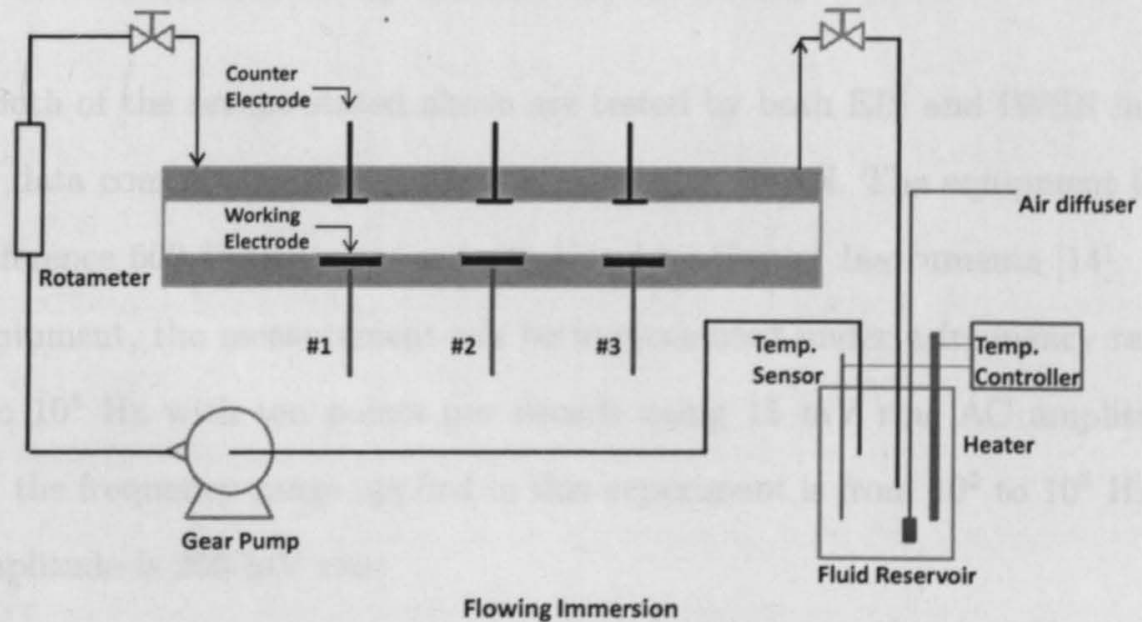

Figure 17. Schematic diagram of flowing immersion [27] 


\subsubsection{Stationary Immersion Setup}

In the stationary immersion setup, there is one O-ring glass electrochemical cell attached to coating panels with a glass tube clamp. The experimental setup is shown in Fig. 18. Cardboard was placed on the rear side of the panel as to insulate the substrate from the clamp. The electrochemical cell is filled with stationary DI water. For the cell, the steel substrate is used as the working electrode. The platinum mesh immersed in the stationary DI water acts as a counter electrode. The wireless sensor is connected to working electrode and counter electrode, and measures the coating impedance.

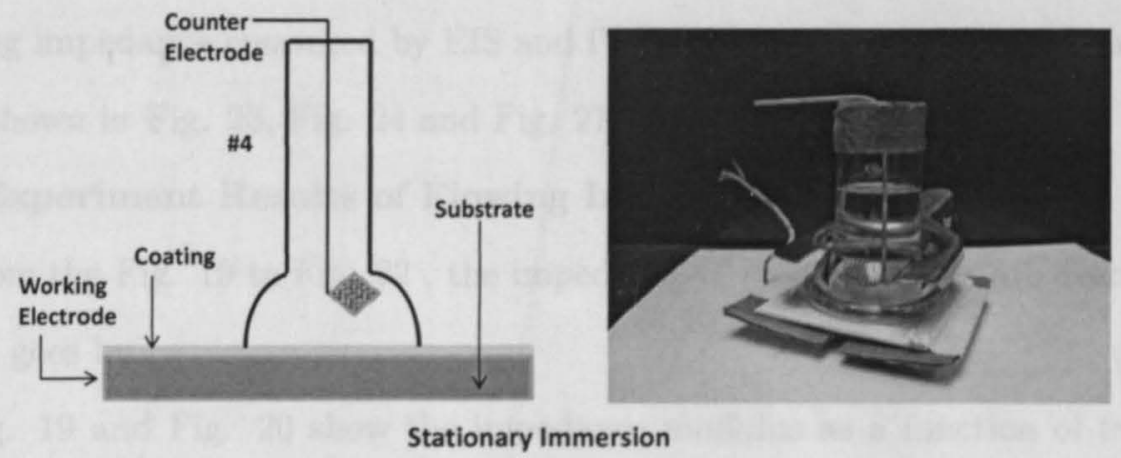

Figure 18. Experimental setup of stationary immersion

Both of the setups stated above are tested by both EIS and IWSN in order to do the data comparison and verify the validity of IWSN. The equipment for EIS is the Reference 600 Potentiostat manufactured by Gamry Instruments [14]. By using the equipment, the measurement can be implemented under a frequency range from $10^{-2}$ to $10^{5} \mathrm{~Hz}$ with ten points per decade using $15 \mathrm{mV}$ rms $\mathrm{AC}$ amplitude. For IWSN, the frequency range applied in this experiment is from $10^{3}$ to $10^{5} \mathrm{~Hz}$ and the AC amplitude is $200 \mathrm{mV}$ rms. 


\subsection{Experiment Results}

EIS data and IWSN data are both collected for coating panels immersed in the flowing fluid as well as the stationary condition in order to demonstrate the coating degradation over 30 days. There are four panels immersed in DI water and three of them are chosen to illustrate the variation of coating impedance. The impedance modulus as a function of frequency as different immersion times for the the selected panels immersed in the flowing fluid is shown from Fig. 19 to Fig. 22. The impedance modulus as a function of frequency as different immersion times for the the selected panels in the stationary condition are shown Fig. 25 and Fig. 26. The comparison of coating impedance measured by EIS and IWSN for panel \#1, panel \#2 and panel \#3 are shown in Fig. 23, Fig. 24 and Fig. 27 respectively.

\subsubsection{Experiment Results of Flowing Immersion}

From the Fig. 19 to Fig. 22 , the impedance of coating panels are decreased as the time goes by.

Fig. 19 and Fig. 20 show the impedance modulus as a function of frequency measured by EIS and IWSN, respectively, for panel \#1. Day1 is the first day that the coating sample was immersed in the DI water. Day4 shows an obvious impedance drop, which means the protection performance of the coating sample has been degraded after 3 days due to the fluid DI water corrosion. As the time goes, the coating impedance keeps dropping. However, the speed of coating impedance decrease is slower than the first few days. This demonstrates that the degradation of coating samples reached the maximum corrosion level.

In Fig. 21 and Fig. 22, the panel \#2 degradation trend is almost the same as panel \#1. In the first few days, the coating impedance decreases quickly. As the time passes, the impedance degradation speed is slower than the previous days. However, the coating impedance of panel \#2 is smaller than the coating impedance of panel 
$\# 1$.

In order to compare the measurement results of EIS and IWSN, Fig. 19 and Fig. 20 are combined with Fig. 23 , and Fig. 21 and Fig. 22 are combined with Fig. 24.

From Fig. 23, the degradation trend of coating is similar between EIS and IWSN. However, the impedance of coating measured by IWSN is larger than the impedance measured by EIS. It is due to the calibration and the limitation of AD5933 as already stated above.

In Fig. 24, the degradation trend of coating is very close. During the first 9 days, the coating impedance measured by IWSN is almost the same as the results measured by EIS. However, the coating impedance measured by IWSN changed slower than the impedance measured by EIS equipment.

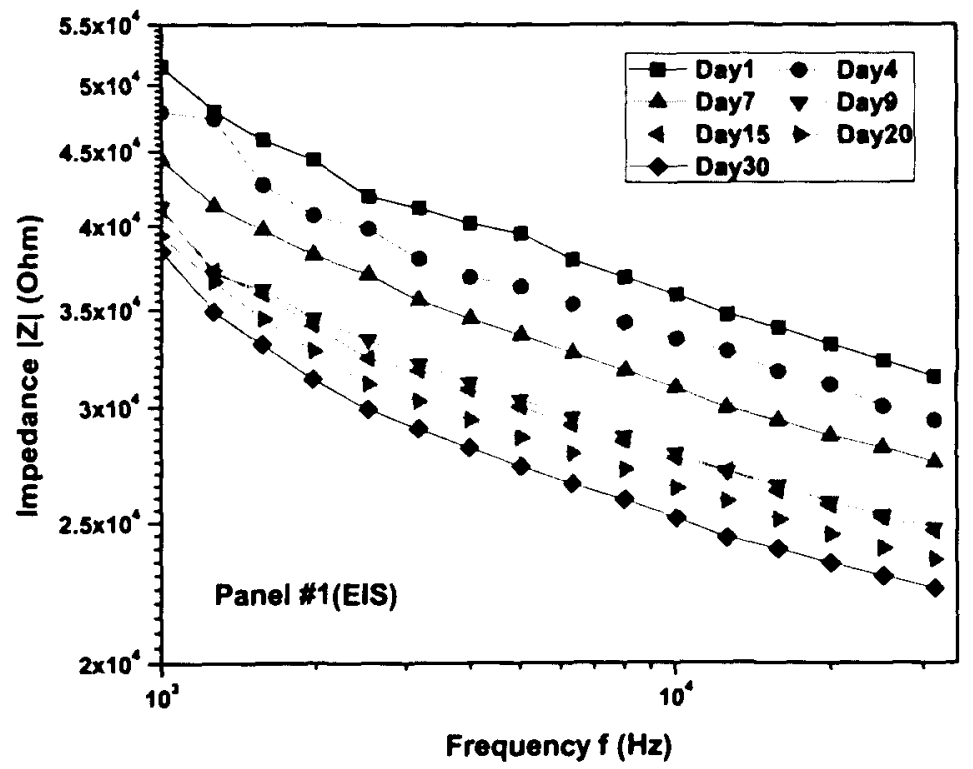

Figure 19. Impedance modulus as a function of frequency at different immersion days for panel \#1 measured by EIS 


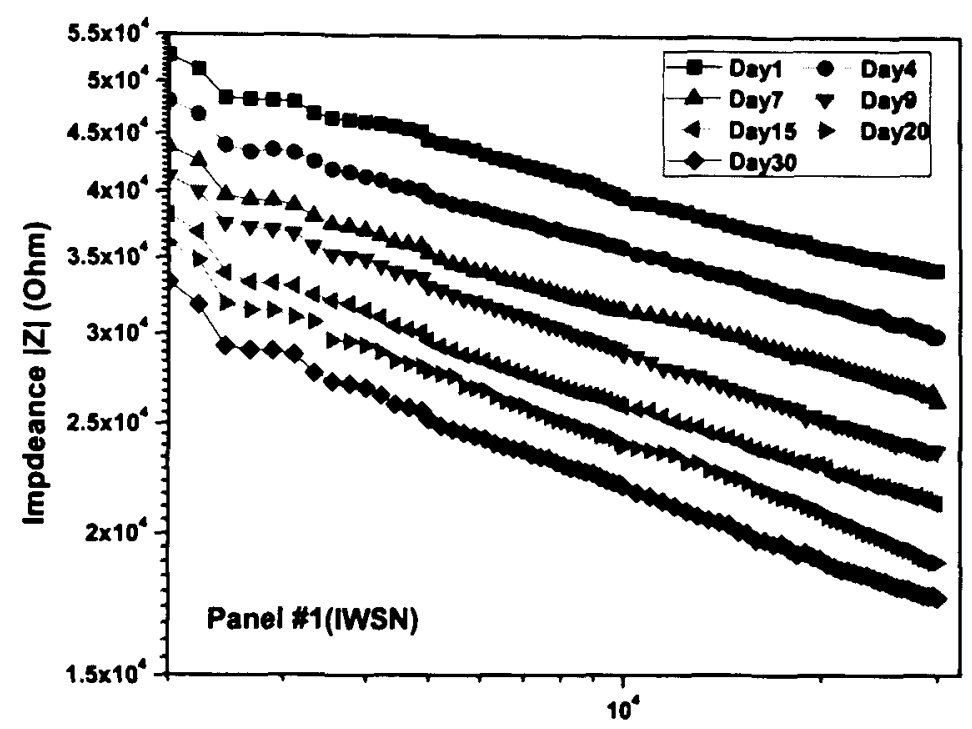

Frequency $f(H z)$

Figure 20. Impedance modulus as a function of frequency at different immersion days for panel \#1 measured by IWSN

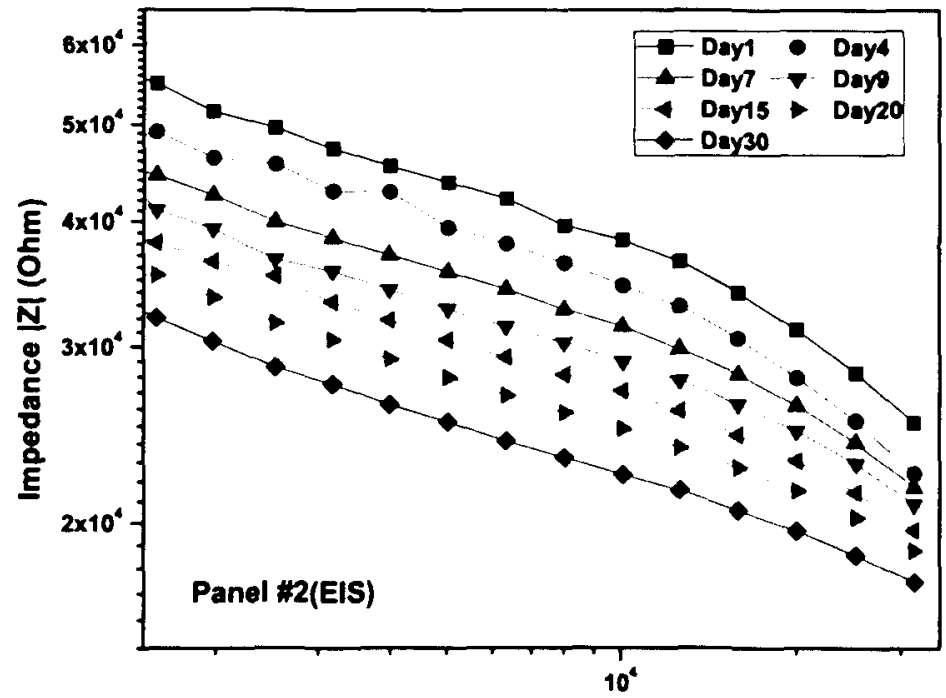

Frequency $\mathrm{f}(\mathrm{Hz})$

Figure 21. Impedance modulus as a function of frequency at different immersion days for panel \#2 measured by EIS 


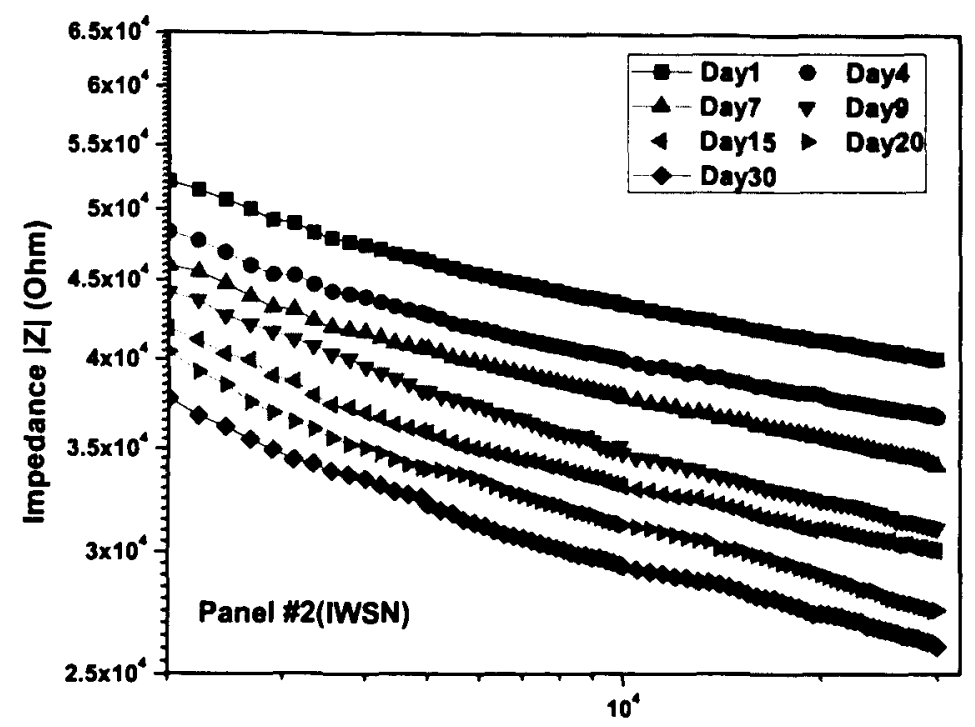

Frequency $\mathrm{f}(\mathrm{Hz})$

Figure 22. Impedance modulus as a function of frequency at different immersion days for panel \#2 measured by IWSN

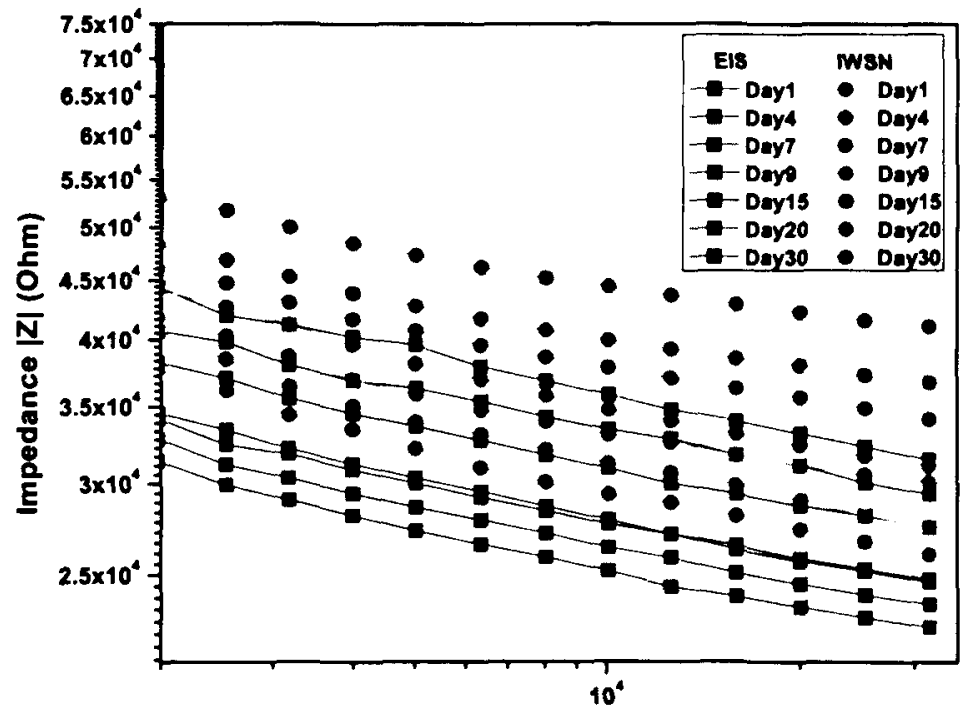

Frequency $\mathrm{f}(\mathrm{Hz})$

Figure 23. Comparison of coating impedance measured by EIS and IWSN for panel $\# 1$ 


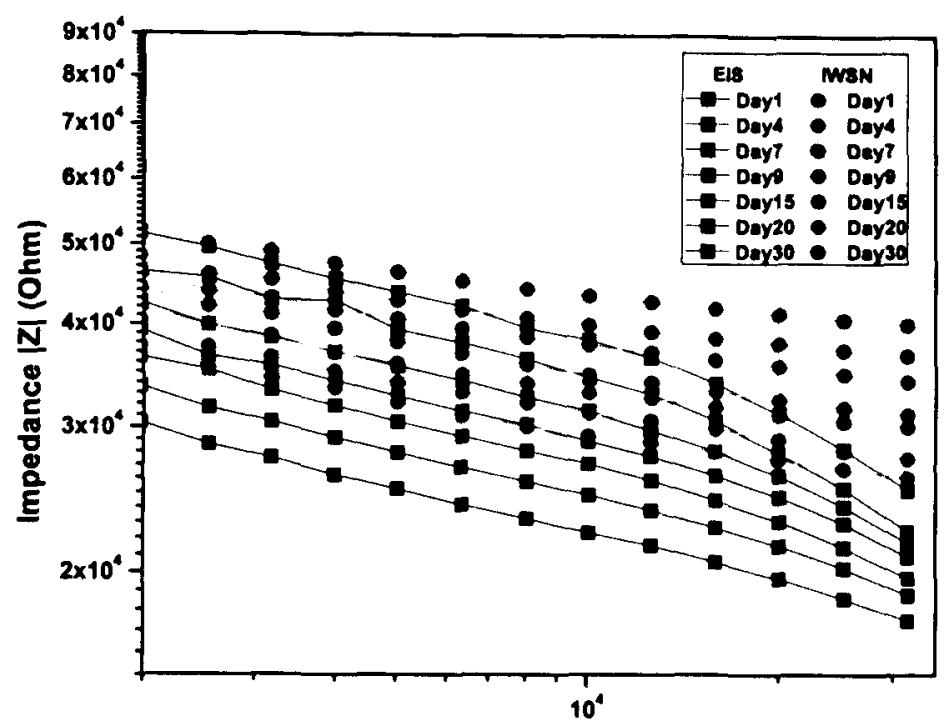

Frequency $f(H z)$

Figure 24. Comparison of coating impedance measured by EIS and IWSN for panel \#2

\subsubsection{Experiment Results of Stationary Immersion}

Fig. 25 and Fig. 26 show the impedance modulus as a function of frequency at different immersion days for panel \#4 immersed in stationary DI water. Compared with panel \#1 and panel \#2, the similar coating degradation is observed. The Day1 impedance is most closed to the original impedance of the coating. After 3 days, there is an obvious impedance drop. However, the coating impedance in the low frequency area decreases slower than the high frequency area. In addition, the impedance modulus decreases as a function of time is more obvious for flowing fluid than stationary fluid. Because of the high quality of the coating samples that don't have pinholes or air bubbles, the decrease of impedance shows that the water uptakes into the coating layer.

Fig. 27 is the combination of Fig. 25 and Fig. 26. The coating impedance 
measured by IWSN is almost the same as the coating impedance measured by EIS equipment. This result can further proves the validity of IWSN in coating evaluation application.

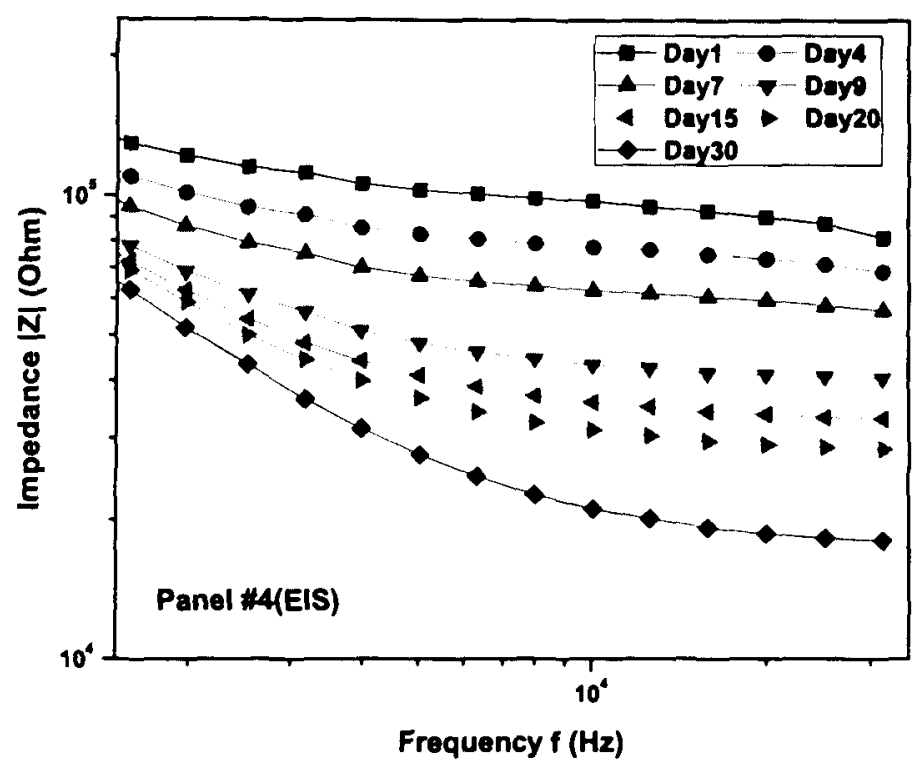

Figure 25. Impedance modulus as a function of frequency at different immersion days for panel \#4 measured by EIS

\subsection{Experiment Summary}

The two experimental results provide good correlations of the impedance modulus as a function of frequency curve under frequency range from $1 \mathrm{KHz}$ to $30 \mathrm{KHz}$. Based on the comparison between the experimental results from the EIS equipment and IWSN , the proposed IWSN is effective in coating evaluation application area. 


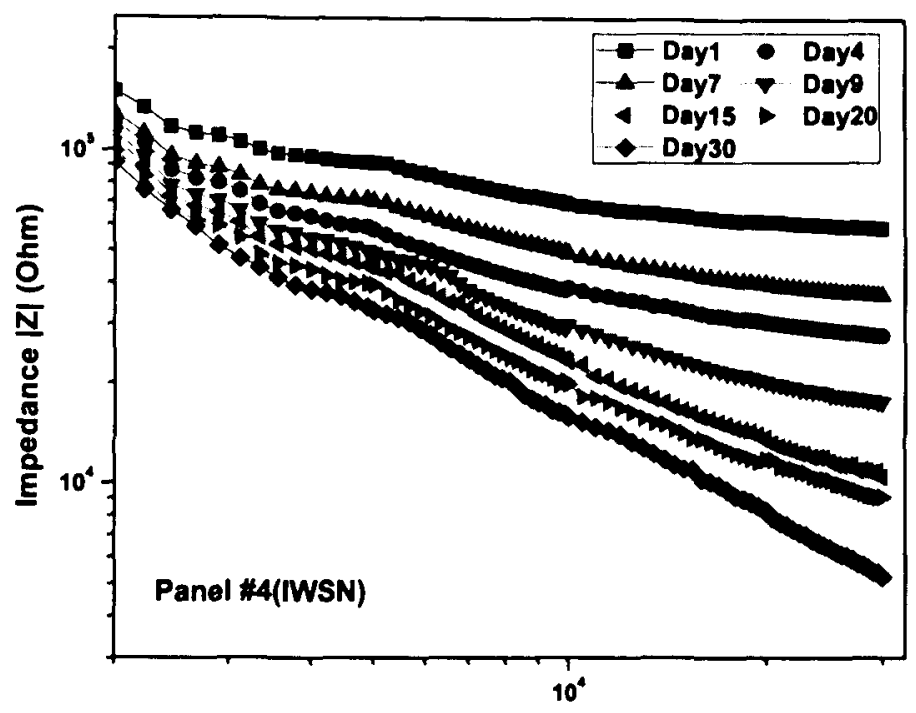

Frequency $f(\mathrm{~Hz})$

Figure 26. Impedance modulus as a function of frequency at different immersion days for panel \#4 measured by IWSN

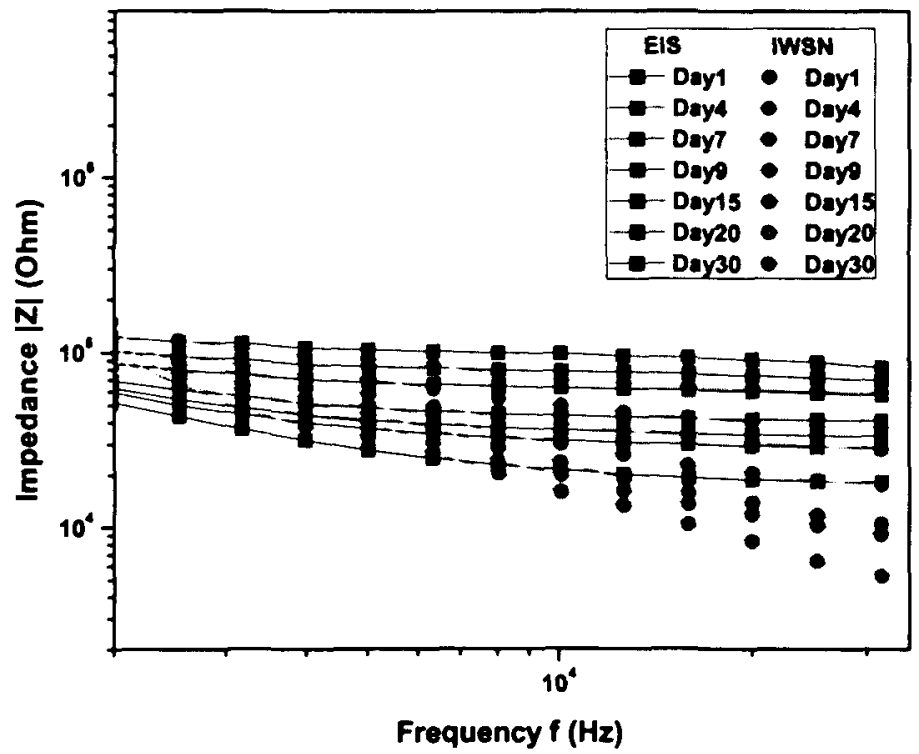

Figure 27. Comparison of coating impedance measured by EIS and IWSN for panel \#4 


\section{CHAPTER 5. CONCLUSION AND FUTURE WORK}

\subsection{Accomplishments}

Coating evaluation is very important in monitoring corrosion degree of the structures and reducing costs associated with corrosion. There are many traditional coating evaluation methods, such as EIS, ENM, and DC resistance. However, all of the coating evaluation equipments based on these coating evaluation methods are bulky, expensive and inconvenient. Therefore, this thesis presented a portable wireless coating evaluation system, IWSN, based on the EIS method. The EIS coating evaluation method measures the coating impedance in the frequency range of 0.01 $\mathrm{Hz}$ to $100 \mathrm{KHz}$, and the coating performance is indicated by the coating impedance variation. The IWSN mainly consists of impedance measurement chip AD5933 and a $\mathrm{RF}$ transceiver $\mathrm{CC} 1111 / \mathrm{CC} 1110 . \mathrm{AD} 5933$ is used to measure the coating impedance and it can be setup through a PC because there is a graphic user interface (GUI) designed by using Visual Basic. The user just needs to click the mouse and select the parameters for coating evaluation. The coating evaluation begins by clicking the "Analysis" button. The wireless sensor nodes start to measure the coating impedance and send the data back to the wireless data base. The wireless data base forwards the data to the PC and the PC plots the coating impedance graph automatically based on the received data. The experimental results measured by EIS instrument (Gamry Reference 600) and IWSN demonstrate the feasibility of IWSN in coating evaluation. The IWSN has much lower cost, power consumption and smaller size compared to Gamry Reference 600 .

\subsection{Contributions}

This thesis contributes to the advanced design of an impedance-based wireless sensor network applied to metal-protective coating evaluation. The contributions of 
this thesis can be divided into three areas: the first design of AD5933 applied on coating evaluation, the design and fabrication of IWSN, and the convenient GUI design.

First, the impedance measurement chip AD5933 was developed by Analog Devices in 2005. After the AD5933 was introduced, there are lots of impedance measurement applications that use the AD5933, especially in Structural Health Monitoring. In 2007, Seunghee Park et al. designed a remote impedance-based loose bolt inspection [22] by using AD5933 and the main application area is the civil infrastructures, such as buildings, bridges, dams, water supply lines, tunnels and etc. In 2008, Fernando Seoane et al applied the AD5933 on biomedical areas [24] to develop portable electrical bio-impedance measurement systems. However, there are no designs applying AD5933 on coating evaluation. Therefore, this IWSN is the first application to use AD5933 on the coating evaluation.

Secondly, the IWSN integrated the impedance measurement single chip AD5933 and the $\mathrm{RF}$ transceiver $\mathrm{CC} 1111 / \mathrm{CC} 1110$. The experimental results obtained by AD5933 are comparable to those obtained by Gamry Reference 600 .

Thirdly, at the PC terminal, a convenient GUI was designed by using VB in order to setup parameters of AD5933, calibration, analysis and plotting. The user can setup parameters easily, such as startup frequency, frequency increment, and sweep points. When the coating analysis is completed, the graphs that plotted by the GUI can be exported by clicking "Save Pics" button.

\subsection{Future Work}

In the future, a number of improvements can be implemented. First, the wireless data base is communicating with the $\mathrm{PC}$ by using serial communication protocol (RS232). In the next design, the RS232 should be replaced by using the USB protocol because the USB is easy to plug in the PC. Actually in the current design, there is 
already a USB interface, but this USB is only used for power supply. In addition, the full speed of the USB2.0 is $12 \mathrm{Mbps}$ and the fastest speed of RS232 is $250 \mathrm{Kbps}$. The USB has obvious speed advantages over RS232. Secondly, it is necessary to reduce the power consumption of the wireless sensor nodes in order to increase the batteries usage time. The power consumption can be reduced by modifying hardware and software design. In hardware, the AD5933 and the RF transceiver can be integrated into one single chip that will reduce the both size and power consumption. In the software, the wireless communication protocol should be optimized in order to ensure each wireless sensor node makes best use of the energy. 


\section{REFERENCES}

[1] V. S. Agarwala, P. L. Reed, and S. Ahmad, Corrosion detection and monitoring - a review, 2000, p. 271.

[2] E. Akbarinezhad, F. Rezaei, and J. Neshati, Evaluation of a high resistance paint coating with EIS measurement: Effect of high AC perturbations, Progress in Organic Coating 61 (2008), 45-52.

[3] K. N. Allahar, D. Wang, D. Battocchi, G. P. Bierwagen, and S. Balbyshev, Real-time monitoring of an air force topcoat $/ \mathrm{mig}$-rich primer system in b11 7 exposure by embedded electrodes, Conference on corrosion science, Atalanta, Geogeia (March 2009).

[4] E. Angelini, A. Carullo, S. Corbellini, F. Ferraris, V. Gallone, S. Grassini, M. Parvis, and A. Vallan, Handheld-impedance-measurement system with sevendecade capability and potentiostatic function, IEEE Trans. On Instrumentation and Measurement 55 (2006), 2.

[5] V. A.Tyagai and N. B. Lukyanchikova, Electrochemical noise of reversible electrode reactions, Electrochimica Acta 18 (1973), 229-230.

[6] G. P. Bierwagen, D. J. Mills, D. E. Tallman, and B. S. Skerry, Proceedings of the conference on electrochemical noise for corrosion applications 1994, ASTM (1996).

[7] G. Blanc, I. Epelboin, C. Gabrielli, and M. Keddam, Electrochemical noise generated by anodic dissolution or diffusion processes, Electroanalytical Chemistry and Interfacial Electrochemistry 75 (1977), 97-124.

[8] Sean Brennan, Measuring a grounded impedance profile using the AD5933, Analog Devices Application Note-847 (2006).

[9] K. D. Conners, W. J. Van Ooij, D. J. Mills, and G. P. Bierwagen, Comparison of electrical impedance spectroscopy and electrochemical noise measurement of plasma polymerized films as pretreatment for coldrolled steel, British Corrosion $35(2000)$.

[10] Analog Devices, AD5933 Datasheet, Available: http://www.analog.com.

[11] N. Fredj, S. Cohendoz, X. Feaugas, and S. Touzain, Some consequences of saline solution immersion on mechanical behavior of two marine epoxy-based coatings, Progress in Organic Coatings 69 (2010), no. 1, 82-91.

[12] L. Fu, K. A. Khor, H. W. Ng, and T. N. Teo, Non-destructive evaluation of plasma sprayed functionally graded thermal barrier coatings, Surface and Coatings Technology (2000), no. 130, 233-239. 
[13] Linda G. S. Gray and Bernard R. Appleman, EIS: Electrochemical impedance spectroscopy: A tool to predict remaining coating life, JPCL (2003).

[14] Gamry Instruments, Evaluation of organic coatings with electrochemical impedance spectroscopy, Available: http://www.labcorr.net.

[15] Texas Instruments, CC1110 Datasheet, Available: http://focus.ti.com.

[16] J. Li and G. P. Biewagen C. S. Jeffcoate, Thermal transition effects and electrochemical properties in organic coatings: Part1 - initial studies on corrosion protective organic coatings, Corrosion Science (1998), no. 54, 763-771.

[17] D. J. Mills, S. Berg, and G. P. Bierwagen, Advances in corrosion protection by organic coatings, Electrochemical Society 95 (1995), no. 13, 82-97.

[18] D. J. Mills and S. J. Mabbutt, Investigation into mechanism of protection of pigmented alkyd coatings using electrochemical and other methods, Progress in Organic Coatings 39 (2000), 41-48.

[19] D. J. Mills, T. Singh, and C. P. Woodcock, Electrochemical methods for examining organic coatings, Progress in Organic Coatings (2011).

[20] Douglas J Mills, Comparison of enm, eis and dc resistance for assessing and monitoring anti-corrosive coatings, Corrosion Science and Engineering 8 (2008).

[21] Gyuhae Park, Harley H. Cudney, and Daniel J. Inman, An integrated health monitoring technique using structural impedance sensors, Journal of Intelligent Material Systems and Structures 11 (June 2000).

[22] Seunghee Park, Chung-Bang Yun, and Daniel J. Inman, Remote impedance-based loose bolt inspection using a radio-frequency active sensing node, Korean Society for Nondestructive Testing 27 (June 2007), 3.

[23] Isao Sekine, Makoto Yuasa, Norimitsu, Hirose, and Toshiyuki Tanaki, Degradation evaluation of corrosion protective coatings by electrochemical, physicochemical and physical measurements, Progress in Organic Coatings (2002), no. 45, 1-13.

[24] Fernando Seoane, Javier Ferreira, Juan Jose Sanchez, and Ramon Brago, An analog front-end enables electrical impedance spectroscopy system on-chip for biomedical applications, Physiological Measurement (2008), no. 29, 267-278.

[25] Texas Instruments SimpliciTi, SimpliciTi Protocol, Available: http://focus.ti.com.

[26] V. A. Tyagai and N. B. Lukyanchikova, Electrochemical noise of iodine reduction on a cadmium sulfide surface, Surface Science 12 (1968), 331-340. 
[27] Y. Wang and G. P. Bierwagen, A new acceleration factor for the testing of corrosion protective coatings: flow-induced coating degradation, J. of Coatings Technology and Research 6 (2009), no. 4, 429-436. 


\title{
APPENDIX A. SOURCE CODE FOR THE WIRELESS SENSOR NODE
}

\author{
\#include "bsp.h" \\ \#include "mrfi.h" \\ \#include "nwk_types.h" \\ \#include "nwk_api.h" \\ \#include "bsp_leds.h" \\ \#include "bsp_buttons.h" \\ \#include "uarto.h" \\ \#include "app_remap_led.h"
}

\#define SPIN_ABOUT_A_SECOND NWKDEI_AY(1000)

static linkID_t sLinkID1 $=0$;

static uint8_t rReceiverData[39];

/* application $\mathrm{Rx}$ frame handler. */

static uint $8_{-} t$ sRxCallback(linkID_t);

static void linkTo(void);

static void linking(void);

void toggleLED( uint $8_{-} t$ );

void main (void)

\{

$$
\begin{aligned}
& \text { BSP_Init (); } \\
& \text { Uart0_Init (); }
\end{aligned}
$$


\#if def I_WANT_TO_CHANGEDEFAULT_ROMDEVICE_ADDRESS_PSEUDO_CODE

\{ $\operatorname{addr} r_{-}$lAddr;

createRandomAddress(\&lAddr);

SMPL_Ioctl (IOCTL_OBJ_ADDR, IOCTL_ACT_SET, \&lAddr);

\}

\#endif

SMPL_Init ( sRxCallback);

/* turn on LEDs. */

if (!BSP_LED2_IS_ON ( ) )

\{

toggleLED ( 2);

\}

if (!BSP_LED1_IS_ON ())

\{

toggleLED ( 1);

\}

/* wait for a button press...*/

do \{

if (BSPBUTTON1() || BSPBUTTON2())

\{ 


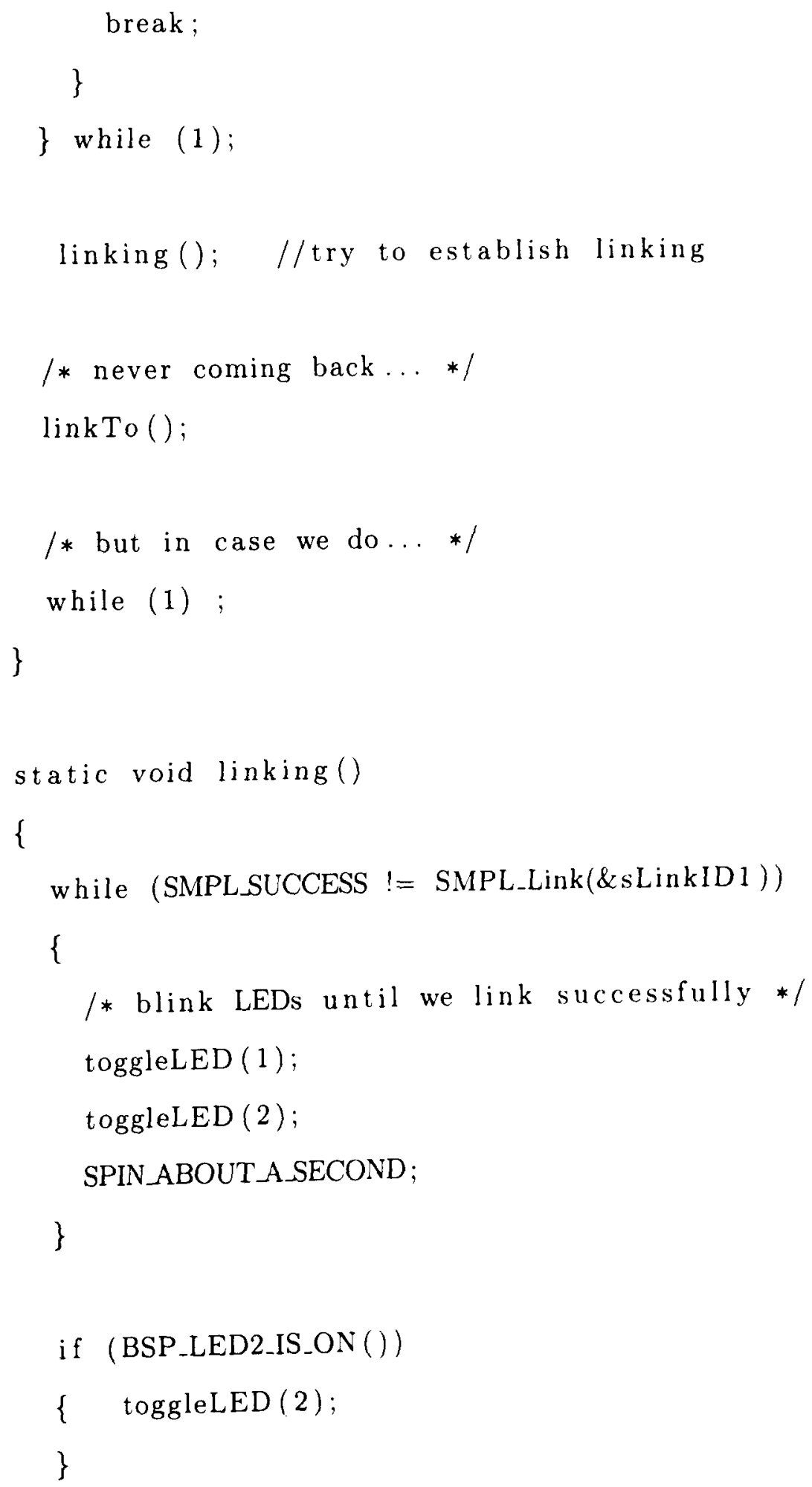




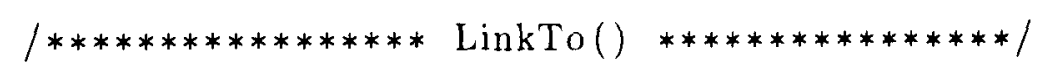

static void linkTo()

\{

uint8_t $\operatorname{msg}[2]$, delay $=0$;

uint $16_{-}$t r Count $=0$;

/* turn on RX. default is RX off.*/

SMPL_Ioctl ( IOCTL_OBJ_RADIO, IOCTL_ACT_RADIO_RXON, 0);

while (1)

\{

SPIN_ABOUT_A_SECOND;

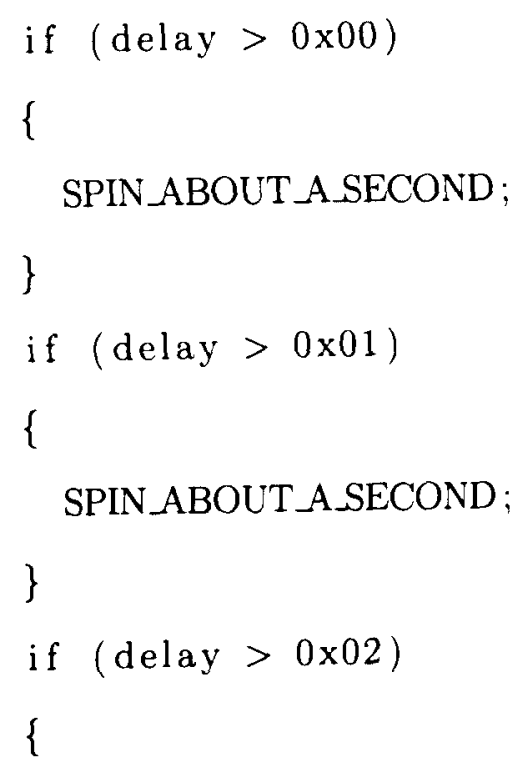


/* delay longer and longer - then start over */

delay $=(\operatorname{delay}+1) \& 0 \times 03$;

/* put the sequence ID in the message */

SMPL_Send(sLinkID1, msg, sizeof (msg));

if $(\mathrm{rCount}>5)$

\{

RS232_SendCharacter (rCharacterArray, sizeof (rCharacterArray)); RS232_CRLF ();

RS232_SendData ();

RS232_CRLF ( ) ;

$\mathrm{rCount}=0$;

\}

$$
\text { rCount }++ \text {; }
$$

\}

\}

void toggleLED ( uint $8_{-}$t which)

\{

$$
\text { if }(1=\text { which })
$$

\{

BSP_TOGGLE_LED1 ( );

\} 


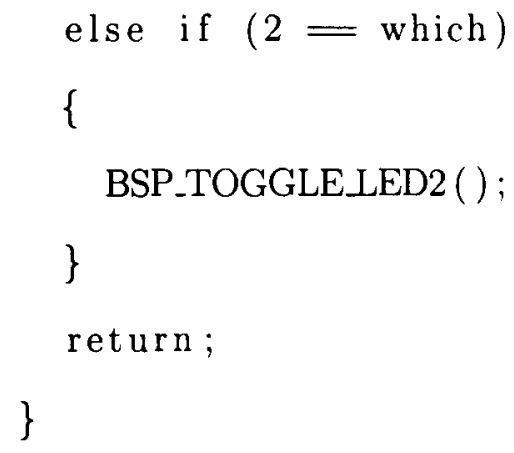


switch ( rReceiverData [0] )

\{

/* Sending "Coating Evaluation is in Processing" */

case 'C':

/* Sending Real Part Data of AD5933 */

case $0 \times 55$ :

RS232_RealHighLowByte(rReceiverData， sizeof(rReceiverData)); break;

/* Sending Imagenary Part Data of AD5933*/

case $0 \times 66$ :

RS232_ImagHighLowByte(rReceiverData, sizeof(rReceiverData)); break ;

default :

break;

\}

toggleLED ( 2);

/* drop frame. we're done with it. */

return 1;

\}

\} 
/* keep frame for later handling. */ return 0 ;

\} 


\section{BASE}

\#include "bsp.h"

\#include "mrfi.h"

\#include "nwk_types.h"

\#include "nwk_api.h"

\#include "bsp_leds.h"

\#include "bsp_buttons.h"

\#include "app_remap_led.h"

\#include "AD5933.h"

static void linkFrom(uint $8_{-}$t *array, uint $8_{-}$ten);

static void linking(void);

void toggleLED( uint 8_t );

static linkID_t sLinkID2 $=0$;

static volatile uint8-t sSemaphore $=0$;

static uint8_t TXData $[21]=\{$ "Coating Evaluation!!" $\}$;

/* Rx callback handler */

static uint8_t sRxCallback(linkID_t);

void main (void)

\{

uint8_t Status; 
BSP_Init ( );

AD5933_Init ();

\#if d ef ILWANT_TO_CHANGEDEFAULT_ROM_DEVICE_ADDRESS_PSEUDO_CODE

\{

add $r_{-} t$ IAddr ;

createRandomAddress(\&lAddr);

SMPL_Ioctl (IOCTL_OBJ_ADDR, IOCTL_ACT_SET, \&lAddr );

\}

\#endif

SMPL_Init (sRxCallback);

/* turn on LEDs. */

if (!BSP_LED2_IS_ON ()$)$

\{

toggleLED (2);

\}

if (!BSP_LED1_IS_ON ( ))

\{

toggleLED (1);

\}

linking (); // try to establish link 


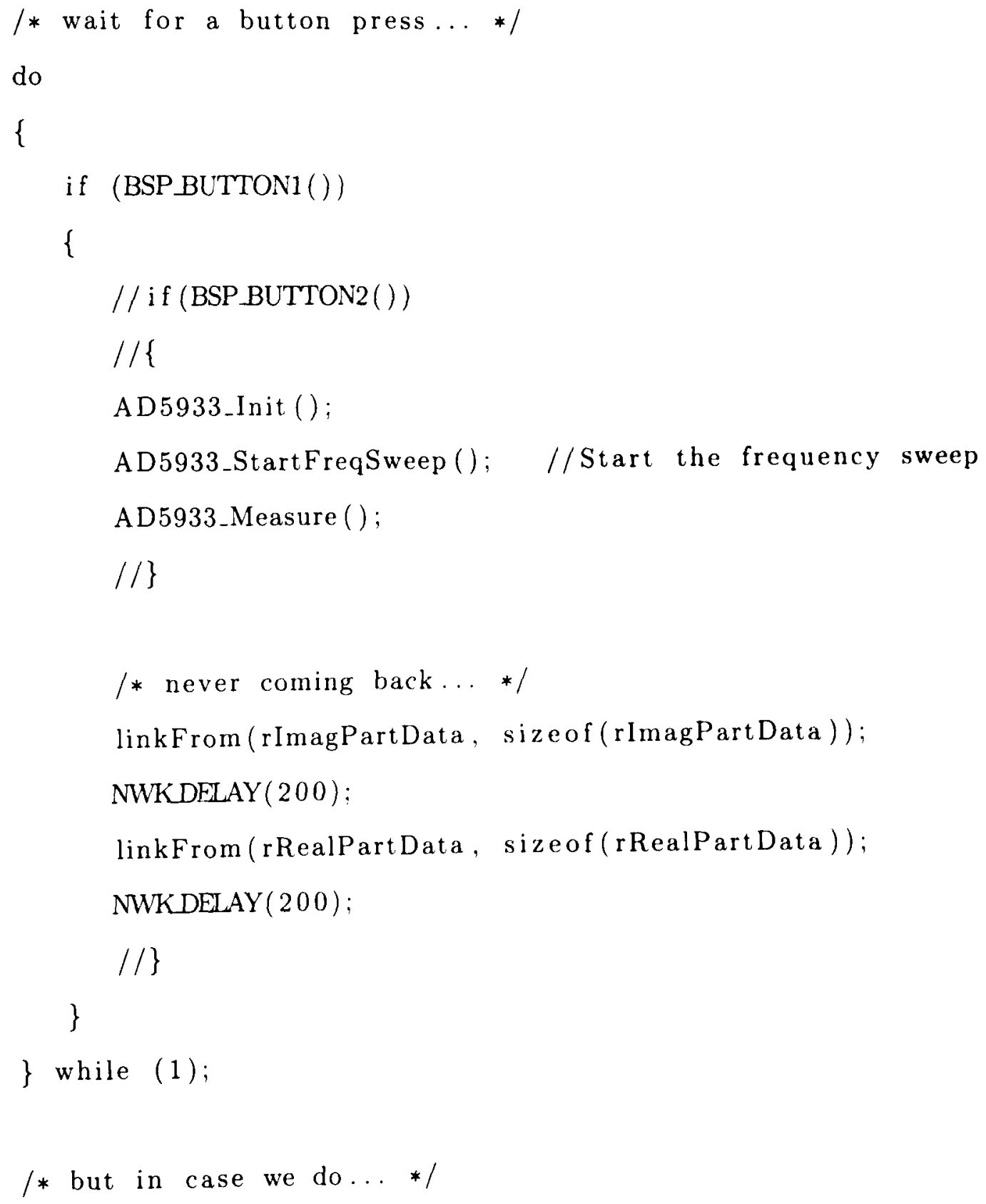




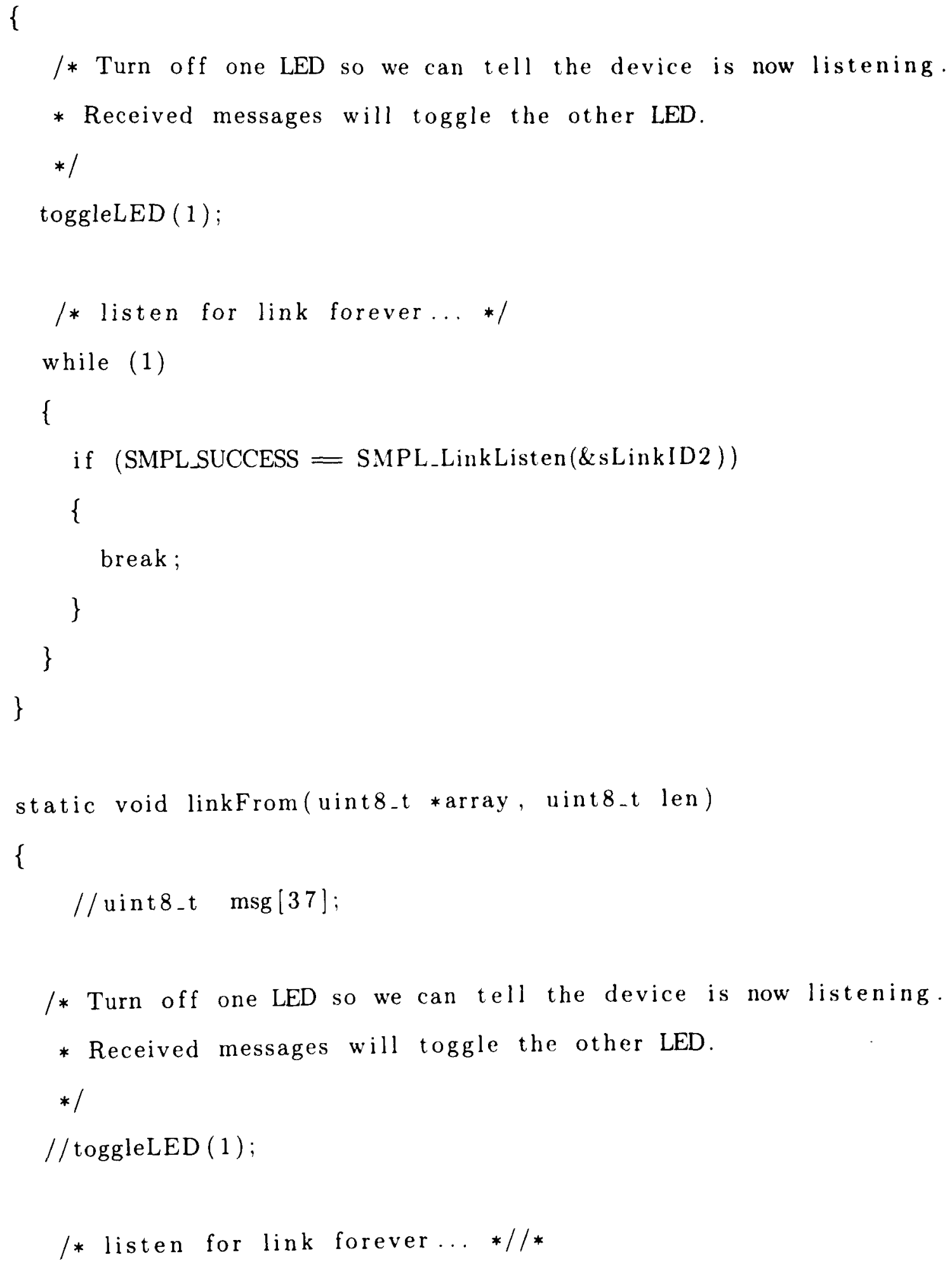


while (1)

\{

if (SMPL_SUCCESS $=$ SMPL_LinkListen(\&sLinkID2))

\{

break;

\}$* /$

/* Implement fail-to-link policy here, otherwise, listen again. */ $/ /\}$

/* turn on RX. default is RX off. */

SMPL_Ioctl( IOCTL_OBJ_RADIO, IOCTL_ACT_RADIO_RXON, 0);

while (1)

\{

if (sSemaphore)

\{

$/ / *(\operatorname{msg}+1)=++\mathrm{tid}$

//SMPL_Send (sLinkID2, msg, 2);

SMPL_Send (sLinkID2, array, len);

sSemaphore $=0$;

break;

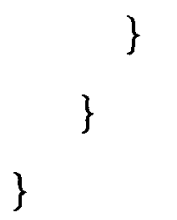

$/ * * * * * * * * * * * * * * * * * * * *$ Toggle LED 
void toggleLED (uint _t $_{\text {t }}$ which)

\{

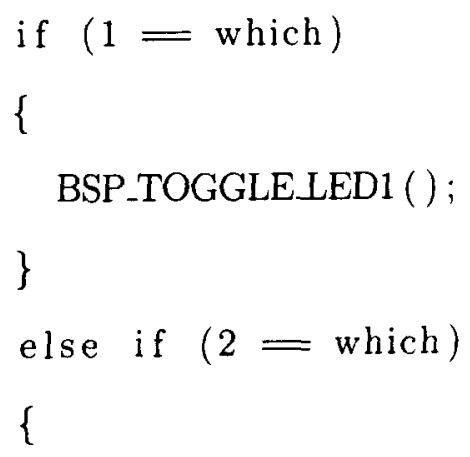


/* Post to the semaphore to let application know so it sends

* the reply

*/

sSemaphore $=1$;

/* drop frame. we're done with it. */

return 1;

\}

\}

/* keep frame for later handling */

return 0 ;

\} 